Visual and motion cue integration in a dynamic motion seat

by

\title{
Peter Townsend
}

A thesis submitted to

the Faculty of Graduate and Postdoctoral Affairs

in partial fulfillment of the requirements for the degree of

Master of Arts

in

Psychology

Carleton University

Ottawa, Canada

(C)2014 Peter Townsend 


\begin{abstract}
The purpose of this thesis was to extend the understanding of how complementary motion and visual cues affect performance in perceptual tasks in dynamic motion seats. In Experiment 1, participants received motion cues from a dynamic motion seat and a visually presented attitude indicator that simultaneously displayed bank angles of $10^{\circ}$, $20^{\circ}, 30^{\circ}$, and $40^{\circ}$. Participants were required to estimate each bank angle from the attitude indicator. The addition of motion cues from the motion seat that were consistent with the attitude-indicator did not improve performance compared to when no motion seat cues were provided. The addition of inconsistent cues from the motion seat reduced estimate accuracy. Experiment 2 was identical to Experiment 1 except that participants were required to estimate bank angles from the motion seat. The addition of consistent visual cues increased estimate accuracy compared to having no visual cues, and inconsistent visual cues decreased estimate accuracy.
\end{abstract}




\section{Acknowledgments}

There are several people who deserve credit for guiding me through the process of completing my thesis.

I would like to thank my supervisor Chris Herdman for the opportunity to be a part of this lab, and to learn the research process under such a successful researcher. I consider myself extremely fortunate to be provided with the resources and guidance needed to complete this project.

Thank you to Matt Brown for the countless hours spent helping me write this thesis and improve my writing skills.

I am grateful to my committee for the invaluable feedback you have provided me with during the research and writing process.

Thank you to Andrew Staples for the technical support. Without you this project would not have been possible.

Finally, I would like to thank my family and friends for the unconditional support and patience while I complete my graduate studies. 


\section{Table of Contents}

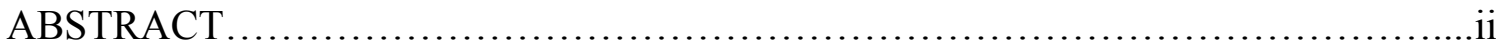

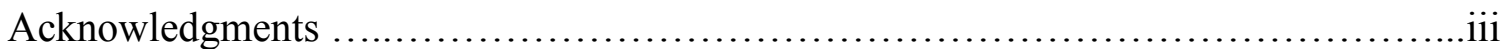

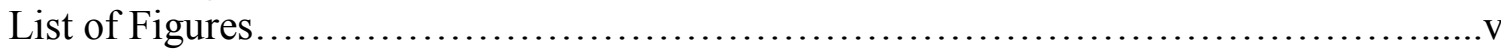

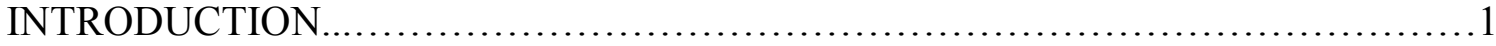

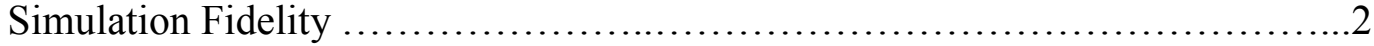

Cognitive and Sensory Research in Aviation Psychology ......................... 4

Motion Cueing - Platform Motion .........................................

Pilots' Subjective Preference for Motion ....................................6

Alternative Motion Cueing …...............................................

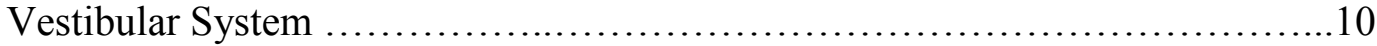

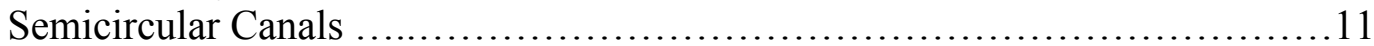

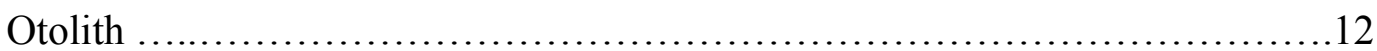

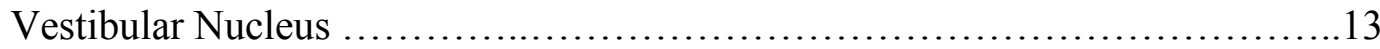

Vestibulo-Ocular Reflexes .................................................

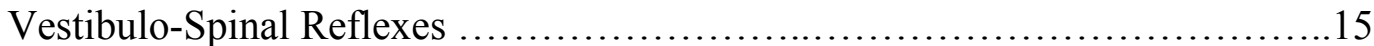

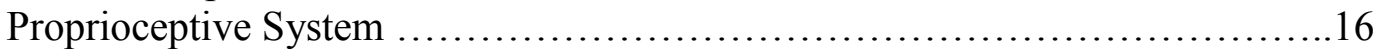

Muscle Spindles ....................................................... 16

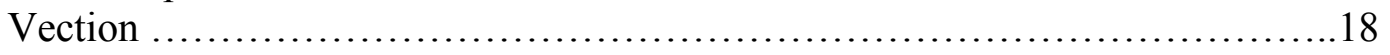

Integration of Visual and Motion Cues .......................................21

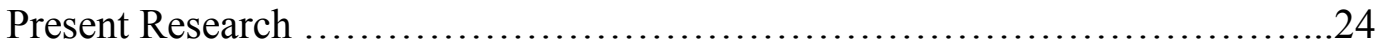

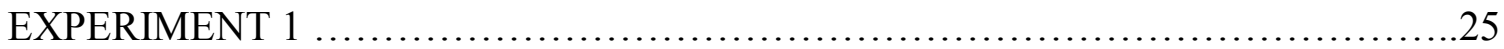

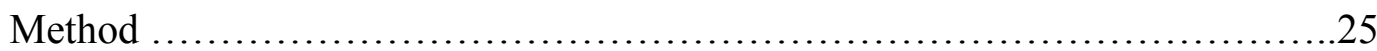

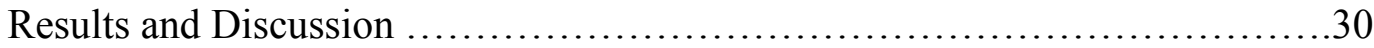

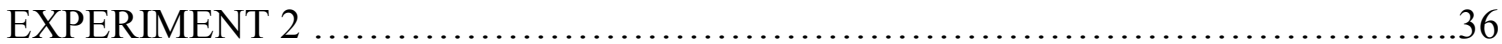

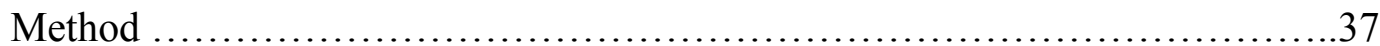

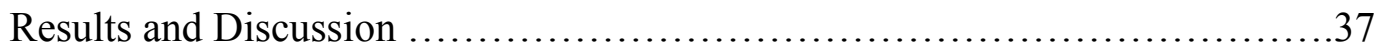

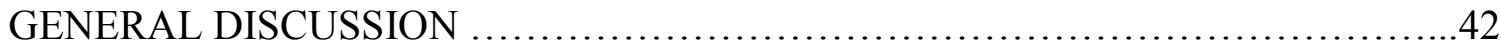

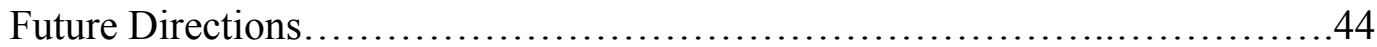

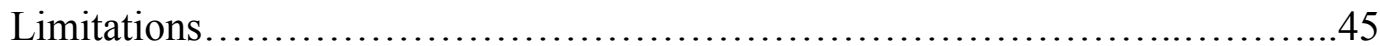

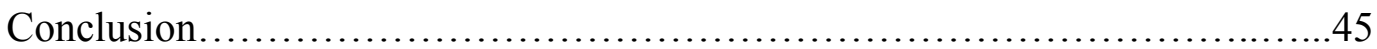

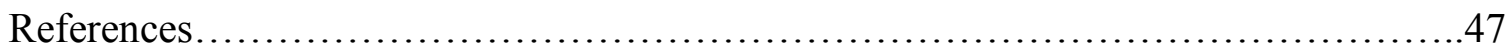

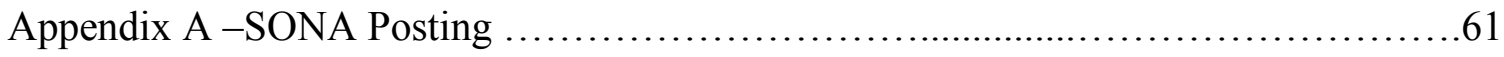

Appendix B -Informed Consent Form ............................................62

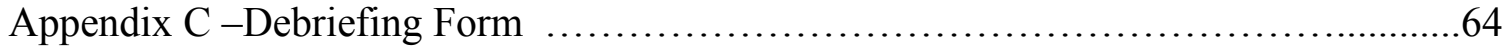




\section{List of Figures}

Figure 1. Illustration of the inner ear. ........................................11

Figure 2. Anatomy of the vestibular system. .................................. 12

Figure 3. Anatomy of a muscle spindle. ..................................... 17

Figure 4. Movement capabilities of a dynamic motion seat. .........................27

Figure 5. Screenshot of an inside-out attitude indicator. ..........................28

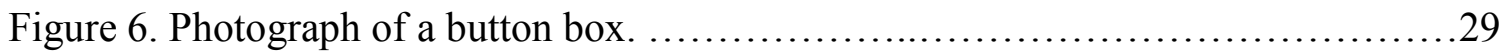

Figure 7. Consistency estimation accuracy as a function of visual bank angle and motion

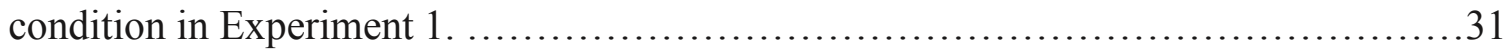

Figure 8. Consistency judgment accuracy as a function of visual and motion-cue disparity

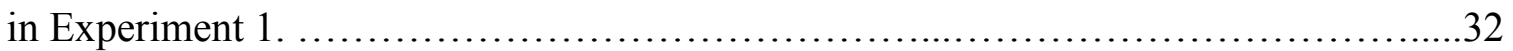

Figure 9. Bank angle estimation accuracy as a function of visual bank angle and motion condition in Experiment 1.

Figure 10. Consistency estimation accuracy as a function of motion seat bank angle and

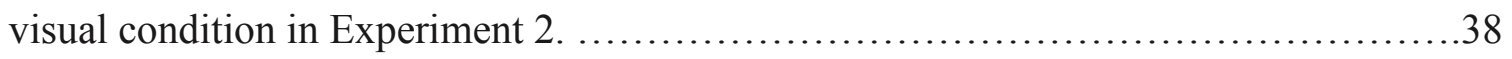

Figure 11. Consistency judgment accuracy as a function of visual and motion-cue disparity in Experiment 2.

Figure 12. Bank angle estimation accuracy as a function of motion seat bank angle and visual condition in Experiment 2. 


\section{INTRODUCTION}

Over the past 30 years, there have been dramatic increases in both the research and application of simulator training in aviation (Klyde et al., 2013). Simulators are used as training devices in aviation because they provide a relatively risk-free means to practice complex, and in some cases, life-threatening procedures (e.g., recovery from an unusual orientation; Lee \& Myung, 2013). Increases in safety awareness within the aviation industry have amplified the demand for simulator training. As a consequence of the growing safety awareness, the number of commercially-available simulators continues to grow as technological developments in simulation technology continue to improve. These developments increase the demand for advanced simulators that can replicate all in-flight scenarios (Maran \& Glavin, 2003). A fundamental question is what degree of precision does a flight simulator need to replicate real-life aviation in order to provide the most effective training and research? The degree of precision with which a flight simulator replicates actual flight is known as fidelity, and has been a topic of debate within the aviation industry for decades (Maran \& Glavin, 2003). Although many aviation researchers and pilots believe that highly precise simulators are needed for adequate flight training, there is little objective evidence to support this belief. Cheaper low-fidelity simulators have been gaining popularity in training research because of the lack of support for the benefits of high-fidelity simulators. This increase in popularity has led to increased research exploring the cognitive and perceptual experiences of operators within low-fidelity simulators. The goal of the present research was to evaluate how motion cues from a low-fidelity motion cueing seat affect operators' ability to perceive their spatial orientation. 


\section{Simulation Fidelity}

Fidelity has been defined as the extent to which the appearance and behaviour of the simulated environment matches the appearance and behaviour of the system that is being simulated. A high level of fidelity presumably equates to a more realistic simulation (Farmer, van Rooij, Riemersma, Joma \& Morall, 1999). Miller (1953) proposed two distinct, complementary categories of simulator fidelity - engineering and psychological. Engineering fidelity, or physical fidelity, is defined as the degree to which the simulated environment replicates the environmental characteristics of the real task. Increasing the engineering fidelity of the simulator inevitably leads to increases in cost. For example, increasing the engineering fidelity of a visual display could involve implementing a display that subtends the entire visual field, with the highest possible resolution and refresh rate. Psychological fidelity, or functional fidelity, is defined as the degree to which the skills and psychological responses in the real task are captured in the simulated task. It is measured by comparing the operator's behavioural and physiological responses during the simulation to completing the same task in the field. A highpsychological fidelity simulator may elicit very similar, or the exact behavioural and/or physiological responses that would be produced by completing the same task in real-life flight. Researchers can gain insights into the psychological fidelity of the simulator by measuring sensory reflexes such as reaction time, and eye movements in response to simulated tasks. This data would then be compared to the same measurements taken during actual flight. If the simulator elicits the same behaviours produced by actual flight, it can be presumed to have high psychological fidelity (Sadowski \& Stanney, 2002) 
Since the introduction of simulators in aviation training research, many researchers have held the assumption that more realistic simulators provide more effective research and training. This assumption was made evident in the early aviation training literature beginning with Adams (1961), who wrote a textbook chapter in support of the highest fidelity of simulation for transfer of training. Several quantitative studies regarding fidelity in transfer of training were conducted by military and aerospace personnel (Cooper, 1963; Bird \& Daniel, 1963; Smedal, Rogers, Duane, Holden \& Smith, 1963) following Adams (1961). The fidelity of the visual and motion systems of training simulators have primarily been the focus of transfer of training research and flight training administration. For example, the degree of fidelity in training simulators is regulated and monitored by the relevant aviation authorities (e.g., FAA in USA and Transport Canada in Canada). These authorities have classified simulators across four levels of fidelity (A, B, C, and D). A and B have basic visual displays, and motion systems with 3-axis motion, while $\mathrm{C}$ and $\mathrm{D}$ have visual displays with highly specific visual parameters and 6-axis motion systems. Currently the FAA and Transport Canada require 6-axis platform motion for the highest level of pilot training and evaluation (Robinson, Mania \& Perey, 2004).

Many aviation researchers have questioned whether the most realistic simulation of a flight task provides the most benefits for transfer of training and pilot performance. Furthermore, are the benefits of high-fidelity simulators worth the high costs associated with purchasing, operating and maintaining the equipment (Salas \& Cannon-Bowers, 2001)? The findings in most transfer of training studies, especially those related to motion cueing, are scattered and conflicting. Woodruff, Smith, Fuller and Weyer (1976) 
explored the effects of simulator training with motion and no motion in a group of beginner pilots and did not find any benefits of adding motion cueing in terms of performance within the simulators and performance in the field afterwards. Three more studies (Martin \& Waag, 1978a, 1978b; Nataupsky, Waag, Weyer, McFadden \& McDowell, 1979) also showed little evidence of a transfer benefit when using motion versus no motion during simulator training. Contrary to the findings of Woodruff et al. (1976), Richard and Parrish (1984) demonstrated a significant benefit of motion cueing in pilot performance during a simulated hover task. Overall, there have been mixed results in support of motion systems benefiting transfer of training in flight simulation.

\section{Cognitive and Sensory Research in Aviation Psychology}

One limitation of transfer of training experiments is that they do not provide an explanation as to why combining some sensory cues (e.g., auditory cues) with a visual scene produce consistent performance benefits, while incorporating other cues (e.g., motion) have shown inconsistent benefits. Recent research has begun exploring basic cognitive and sensory processes that play an underlying role in how humans perceive sensory cues provided by simulators. Of the many perceptual and cognitive processes, multisensory integration seems to be a highly studied area of interest.

Wickens, Small, Andre, Bagnall and Brenaman (2008), among other aviation researchers (Lam, Mulder \& van Passen, 2007; Eriksson, 2009), have explored cues across sensory systems in their research in order to examine fidelity from a cognitive/sensory perspective. Wickens et al. (2008) compared pilot performance in recovering from an unusual pitch with the use of auditory and visual cues versus tactile and visual cues versus only visual cues. Wickens et al. (2008) found that having 
multisensory cues significantly improved pilots' response times to the unusual pitch compared to purely visual cues. Many studies within aviation psychology have shown that simulators that provide multisensory cues tend to enhance performance in simulator training (Sarter, 2000; Tannen et al., 2004). This is especially the case when simulators provide sensory information that includes adding auditory cues (Tannen et al., 2004), and tactile cues (Wickens et al., 2008) to a visual display. One exception to this observation seems to be simulators that combine visual stimuli with full-platform motion, which has shown inconsistent evidence supporting these complementary sensory cues (McCauley, 2006).

\section{Motion Cueing - Platform Motion}

Motion platforms can move in several combinations of translational and/or rotational axes. Translational degrees of freedom can include surge motion (forward and backward), sway motion (side to side) and heave motion (up and down), while rotational degrees of freedom can include bank motion (tilting of the horizontal axis side to side), pitch motion (tilting of the vertical axis forward and backward), and yaw motion (a movement of the nose from side to side) (Sears \& Jacko, 2009). Fidelity in motion platforms is generally measured using recordings of all variables of motion within the airplane simulator. These measurements are then compared with corresponding measurements from the pilot's seat in an actual airplane (Ashkenas, 1985). High-fidelity motion platforms must be able to simulate all six forms of motion (hence 6 degrees of freedom) and precisely replicate all movements perceived in an actual airplane.

A common 6 degree of freedom motion platform consists of a frame with six or more extendable actuators connecting a fixed hexagon-shaped base to a moveable 
platform. The multiple actuators extend and contract in order to provide specific types of motion. Six degree of freedom motion platforms are the most commonly used motion systems for flight simulators due to the FAA requirements for high-fidelity motion to be used for pilot training and evaluation (Burki-Cohen et al., 2009). A 6 degree of freedom platform on its own does not necessarily provide high fidelity motion; however, when paired with an appropriate flight model and visual display, 6 degree of freedom platforms can effectively provide high fidelity motion. Although the benefits of high fidelity motion have not been consistently demonstrated objectively, most expert pilots subjectively prefer motion to no motion in flight simulators (McCauley, 2006).

\section{Pilots' subjective preference for motion}

From the 1970's through the 1990's, many aviation psychology researchers have included questionnaires examining pilots' subjective preferences of the simulators being used in their experiments. Caro, Jolley, Isley and Wright (1972) reported that pilots prefer motion cues in simulation, presumably because motion cues increase how realistic the simulated flight tasks feel. The authors also noted that more experienced pilots might require more sophisticated motion systems because they would be capable of detecting more subtle differences from the aircraft motion as opposed to novice pilots. Parrish, Houck and Martin (1977) reported an investigation of motion in a helicopter simulator. They found that pilots performing slalom manoeuvres preferred motion, but objective performance measures indicated no advantage of the motion compared to no motion. Parrish et al. (1977) found that pilots tended to control the simulated helicopter differently between the motion and no motion conditions. In the motion condition, the way in which the pilots performed the slalom task more closely resembled the way it 
would be completed in an actual helicopter, compared to the no motion condition. This difference in behavior supports the pilots' subjective preference and suggests a greater level of psychological fidelity in this particular study despite showing no objective performance benefits of motion.

An important series of experiments was conducted that explored whether precisely simulated motion was needed in order to achieve the naturalistic control behaviors and subjective pilot preference found in Parrish et al. (1977). Reid and Nahon (1988) compared a no-motion condition to several different motion algorithms on a highfidelity motion platform, while Bussolari, Young and Lee (1988), and Lee and Bussolari (1989) compared no-motion to high-fidelity motion, 2-degrees of freedom motion, and a 1-degree of freedom heave motion. In all three studies it was found that most pilots disliked the no-motion conditions, while showing no preference differences between any of the motion conditions, and no performance differences amoung all conditions. This finding is distinct from Parrish et al.'s (1977) findings given that a very limited amount of motion was found to be acceptable to pilots. Parrish et al. (1977) only demonstrated that pilots' consistently disliked the extreme case of no motion compared to the other extreme case of full platform motion. The findings of Reid and Nahon (1988), Bussolari et al. (1988) and Lee and Bussolari are consistent with an interpretation that low-fidelity motion may be sufficient to contribute to flight training. These investigations of pilot preference do not provide information about training transfer. However, as McCauley (2006) noted, in decisions about whether or not to purchase, maintain, and operate a motion system, pilot preference and acceptance of the simulator are legitimate factors to be considered, independent from transfer of training. 


\section{Alternative motion cueing}

The use of high-fidelity motion systems comes at a high cost. Bartel and Foster (2004) estimated that the cost of a 20-year life cycle of one high-fidelity motion platform with hydraulic actuators would total $\$ 1.32$ million US, while a high-fidelity simulator with electric actuators would total \$0.93 million US. These estimates included procurement, maintenance, and electricity but did not include costs for the construction and maintenance of a facility that can accommodate the size and weight of the system, nor did the estimates include the cost of labour associated with running the system. Thus, the actual cost of using a high-fidelity motion system may be much greater than estimated (Burki-Cohen, Sparko \& Go, 2007).

Given the high price of operating a high-fidelity motion system, researchers have explored the option of using alternative, lower-fidelity motion systems that come at a lower cost (Burki-Cohen et al., 2007). Dynamic motion seats were explored as a possible alternative to platform motion in early motion fidelity research (Lee \& Bussolari, 1989); however, technological limitations such as limited degrees of freedom (traditionally, motion seats only functioned along the vertical and longitudinal axes) prevented their widespread acceptance. Recent advances in motion technology have allowed manufacturers to address many of these limitations, which has led to a resurgence of dynamic motion seats in aviation training research (Pasma, Grant, Gamble, Kruk \& Herdman, 2011). Empirical findings suggest that pilots' performance and subjective acceptance for motion-cueing seats is similar to that of full-platform motion (BurkiCohen et al., 2009). Grieg (1996) performed a study using a multi-axis dynamic cueing seat in a helicopter-training simulator. Subjective pilot ratings indicated that the addition 
of multi-axis dynamic motion seats to fixed base rotary wing training devices produced significant improvements in simulation realism, and (subjectively) provided a more realistic cognitive workload.

By implementing precision drive motors coupled with bellcranks and push-pull linkages, the performance of modern dynamic motion seats has greatly improved from the early motion seats. Furthermore, modern dynamic motion seats operate in more than just vertical and longitudinal axes. Current dynamic motion seats provide motion in five independent axes of control using four axes of movement: vertical, longitudinal, lateral and roll. (Sutton, Skelton \& Holt, 2010).

Grieg (1996), Burki-Cohen et al. (2009), and Bussolari et al. (1988) explored pilots' subjective acceptance of dynamic motion seats as an alternative to high-fidelity motion. Given the encouraging results of these studies, it seems important to conduct further objective research in understanding how motion cues from dynamic motion seats affect and are perceived by human operators. Nicholson, Townsend, Staples, Gamble and Herdman (2012) conducted a study exploring the effects of congruency of motion cues (provided by a dynamic motion seat) on participants' ability to recover from a simulated disturbance along the roll axis. Nicholson et al. (2013) did not find any significant improvements in performance between a no-motion and a congruent-motion condition (visual disturbance cue and motion cue bank in the same direction). However, they found that participants committed significantly more control reversals (initially corrected in the wrong direction) and over-corrections when presented with incongruent motion cues. The finding of increased control reversals is consistent with Burki-Cohen et al. (2009), as vestibular inputs are generally perceived before visual inputs. A significantly larger 
amount of control reversals within the incongruent condition demonstrates that participants were initially perceiving and reacting to motion cues from the dynamic motion seat before they were able to perceive the direction of the visual stimulus. Although the results of Nicholson et al. (2013) are encouraging, no performance enhancements were found from combining congruent motion and visual cues. Because sophisticated dynamic motions seats have only recently been developed (Sutton et al., 2010), research using these systems is in its infancy. It is important to continue to develop our understanding of how motion cueing from dynamic motion seats affects operators' sensory systems (primarily the vestibular system) and how these cues are perceived, in order to find a low-cost alternative to platform motion systems.

\section{Vestibular system}

The vestibular system is the non-auditory portion of the inner ear that plays a primary role in sensing motion and perceiving spatial orientation of the head. This system is sensitive to the magnitude and angular motion of head movements as an individual rotates or translates (moves in a linear direction, without rotation) through space (Moore, Hirasaki, Raphan \& Cohen, 2001). The sensory information is then carried through the central nervous system and to the vestibular nuclei where it is processed and then combined with spatial information collected by other sensory systems (Cohen, Maruta \& Raphan, 2001). Two separate but complementary organs that are sensitive to different types of head movements collect vestibular sensory information. These organs are the semicircular canals that are responsive to rotational movements of the head, and the otolith, which are responsive to translational movements and forward and backward acceleration (Camis \& Creed, 1930). 


\section{Semicircular Canals}

The inner ear is equipped with three interconnected tubes (known as the semicircular canals) that are each responsible for detecting separate directions of head rotations. These three canals include the horizontal canal, which senses movement on a vertical basis, as the head rotates up and down on the neck, the superior canal, which detects head rotations on the anterior-posterior axis, and the posterior canal, which detects rotations on the sagittal plane. Each canal is partially filled with fluid known as endolymph and is equipped with a dome-shaped structure, called the ampullary cupula, which houses bundles of hair cells called cilia. When the head rotates in a specific orientation, the endolymph of the corresponding canal will flow through the canal, which displaces the cupula and cilia within it (see Figure 1). In contrast, linear accelerations of the head produce equal forces on the two sides of the cupula, which does not create a displacement. The movement of the cilia modulates the receptor potential of the hair cell, which transduces the motion signal of the endolymph into electrical signals that are sent

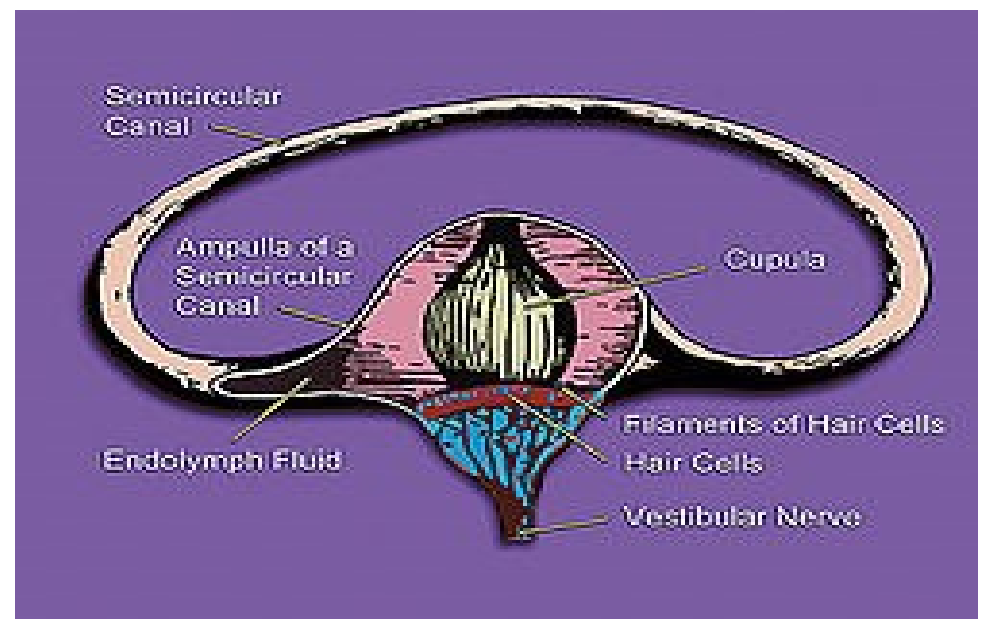

Figure 1. Inner ear illustration showing the semicircular canal, hair cells, ampulla, cupula, vestibular nerve and endolymph. Retrieved May 13, 2014 from http://www.cami.jccbi.gov/aam-400/phys intro.htm. 
through the vestibular nerve and initially to the vestibular nucleus of the brain stem to be decoded by neurons (Rabbitt, Damiano \& Grant, 2004).

\section{Otolith}

The otolith organs are located proximal to the ampullary culpa of the semicircular canals within the inner ear (see Figure 2), and are responsive to translational accelerations commonly produced by inertial or gravitational force. The utricle and saccule are the two main otolith organs. These organs consist of gravity crystals, or otoconia, which are embedded in a gelatinous membrane that also encapsulates cilia that are fixed relative to the skull. The otoconia becomes displaced within the gelatinous membrane during translational accelerations, which then bends the cilia. Much like the cilia of the semicircular canals, the bending of the otolith cilia creates electrical impulses,

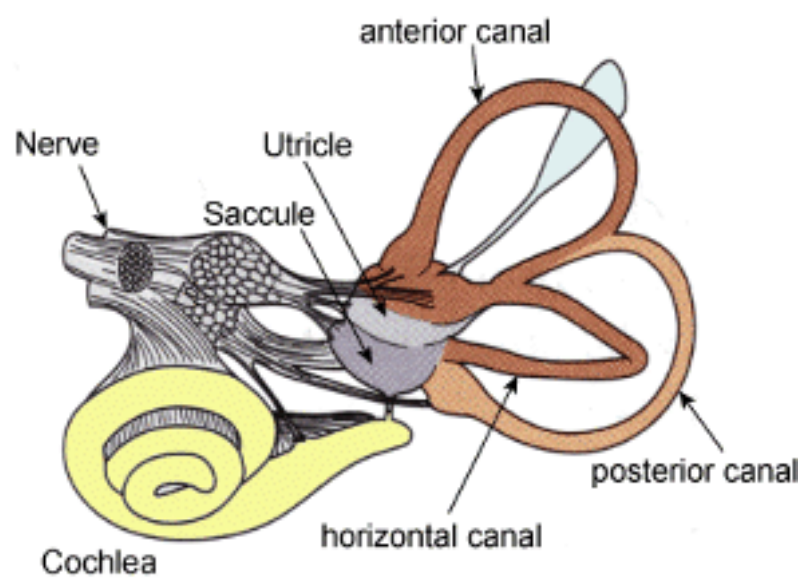

Figure 2. Illustration of the anatomy of the vestibular system within a single ear. Retrieved May 13, 2014 from http://www.answers.com/topic/vestibular-system-1.

which are then projected to the vestibular nucleus (Rabbitt et al., 2004). Signals from the utricle tend to elicit eye movements as opposed to signals from the saccule, which mainly project to the motor cortex areas that control posture. The way in which the brain interprets signals from the otolith is complex, and not yet fully understood. Because 
gravity is equivalent to a constant translational acceleration, the brain must be able to distinguish between gravitational pull and translational movements that are not caused by gravity. Schöne and Udo De Haes (1968) have shown that humans can distinguish between gravitational pull and non-gravitational translational movements quite well, the neural mechanisms that allow for these distinctions however, are not yet fully understood (Cian et al., 2014).

\section{Vestibular Nucleus}

Once the cilia of the semicircular canals and/or otolith have transduced vestibular stimulation into electrical impulses, the electrical signals move through the vestibular nerve and into the brain stem to an area known as the vestibular nucleus (Wilson \& Melvill Jones, 1979). The vestibular nucleus is the primary processor of vestibular input and consists of four major regions, the medial, superior, lateral, and inferior. They are located under the floor of the fourth ventricle and extend from the rostral medulla to the caudal pons in two parallel columns (Khan \& Chang, 2013). The medial vestibular nucleus is the largest and makes up the medial column. The lateral column consists of the superior, lateral, and inferior vestibular nuclei. The medial and superior vestibular nuclei receive input from the semicircular canals and send signals to the motor nuclei of the extraocular muscles in order to stabilize the eyes on a visual target while the head is moving (vestibulo-ocular reflex). The medial nucleus also sends input to the cervical spinal cord in order to coordinate movements between the head and neck (vestibulospinal reflex) (Khan et al., 2013). The lateral vestibular nucleus receives signals from the cerebellum, the semicircular canals, and otolith organs and sends input to the spinal cord. The primary function of this nucleus is to coordinate reflexive tone in the trunk muscles 
and limbs in order to maintain balance and posture. The inferior vestibular nucleus receives information from the otolith organs and then projects to the other vestibular nuclei as well as the cerebellum (Khan et al., 2013). Input from the inferior vestibular nucleus is used by the cerebellum to monitor vestibular performance and to readjust reflexive muscles during changes in acceleration. The cerebellum plays a primary role in updating vestibular information in order for the brain to accurately perform the vestibuloocular and vestibulospinal reflexes.

\section{Vestibulo-Ocular reflexes}

During gaze shifts, in which the eyes and head are oriented to a new visual target, the eyes generally reach the target by a rapid saccadic movement followed by a head turn toward the target. In order to maintain the eyes on the target position during the turning of the head, the eyes must turn in the opposite direction of the head turn at the same rate. This reflex is know as the vestibulo-ocular reflex, and is a product of the interaction of the visual and vestibular systems (Lackner \& DiZio, 2005). Once the eyes are fixed on the visual target, the vestibulocollic reflex stabilizes the head in the desired position if an environmental force imposes additional body movements. Ebenholtz and Shebilske (1975) have shown that even in the dark, with no visual cues presented, the vestibuloocular reflex produces an involuntary pattern of eye movements that reposition the eyes when the body is presented with angular accelerations. However, during constant body rotation in the dark, the semicircular canal signals decay and the involuntary eye movements will gradually stop. In contrast to rotation in the dark, if vision is allowed, the presented rotation will continue to drive the vestibulo-ocular reflex (Lackner et al., 2005). Compensatory eye movements induced by linear acceleration are dependent on the 
orientation of the presented motion and include ocular counter-rolling of the eyes when the body is tilting (Miller \& Graybiel, 1962), ocular counter-rolling during head pitch (the doll's eye reflex; Ebenholtz \& Shebilske, 1975), and elevations and depressions of the eyes during exposure to decreases and increases in background gravitoinertial acceleration during linear translations of the body (Lackner et al., 2005). The vestibuloocular reflex has also been shown to be affected by the visual aspects of a target. For example, when an individual is undergoing left-right translational movements, the amplitude of lateral eye movements rapidly increases when the visual target becomes closer in distance, as would be required to prevent losing sight of the closer object (Telford, Seidman \& Paige, 1997).

\section{Vestibulo-Spinal reflexes}

Vestibular information is used for postural control, particularly in the case of gravitational force (Lackner et al., 2005). Antigravity reflexes are key to standing upright without constant conscious awareness of body position. Reflexes related to standing, balance and body position are usually generated by a complex interaction of the visual, vestibular and proprioceptive systems. For example, when a pilot is presented with motion in the yaw axis, they will first experience a vestibulo-ocular reflex of counterrolling of the eyes and then the head, in the opposite direction of the yaw motion. This reflex is then followed by the corresponding counter-rolling of the shoulders, and other task appropriate body parts (Wilson et al., 1979). The lateral vestibular nucleus is the primary area in facilitating the vestibulospinal reflex. Translational signals from the otolith organs are carried from the lateral nucleus through the lateral spinal tract and projected to each level of the spinal cord (Khan et al., 2013). Rotational signals from the 
semicircular canals are projected to the medial vestibular nucleus and then through the medial vestibulospinal tract to motor neurons that coordinate head and neck movements (Khan et al., 2013).

\section{Proprioceptive system}

The proprioceptive system consists of the interconnection of the spinal cord, muscles and joints, and allows organisms to sense the relative position of neighbouring parts of the body and strength of effort being employed in movement. The proprioceptive system is the primary sense that allows organisms to navigate through the space around them and react rapidly to changing environments, which happens through the displacement of its receptors (Proske \& Gandevia, 2012). Proprioceptive receptors, known as proprioceptors, are found throughout the body, within the skin, joints, and muscles, and commonly interact with one another before their sensory information reaches the brain. For example, the displacement of the knee is commonly accompanied by the displacement of surrounding skin cells and leg muscles, which all contribute information to the corresponding cortical areas (Proske \& Gandevia, 2012).

\section{Muscle Spindles}

Muscle spindles have recently been accepted by most physiologists as the primary proprioceptor, as opposed to proprioceptors within the joints and skin (Proske \& Gandevia, 2012). Muscle spindles are composed of two types of nerves, which are called primary and secondary endings. Primary endings respond to changes in a muscle's length due to stretching, and the speed at which the change in length occurs (Matthews, 1972). Primary endings are therefore believed to contribute both to the sense of limb position and movement velocity. Secondary endings are found within the same muscle fibers but 
are not sensitive to the velocity of muscle length change. Secondary endings signal only the length change itself, so contribute only to the sense of position and not muscle movement (Proske \& Gandevia, 2009). Once stimulated, these nerve endings transduce the kinesthetic energy from the moving muscle into electrical impulses, which are then projected to the spinal cord through primary and secondary sensory fibers (see Figure 3) and then to the brain for processing. Electrical impulses for limb position and movement velocity project through the spinal cord and into the brain (Proske \& Gandevia, 2009). Information regarding limb position and movement velocity project to the premotor cortex, which is a site of convergence of visual, tactile, and proprioceptive information; it is also involved in the control of movement of the mouth, neck, and limbs. Within the

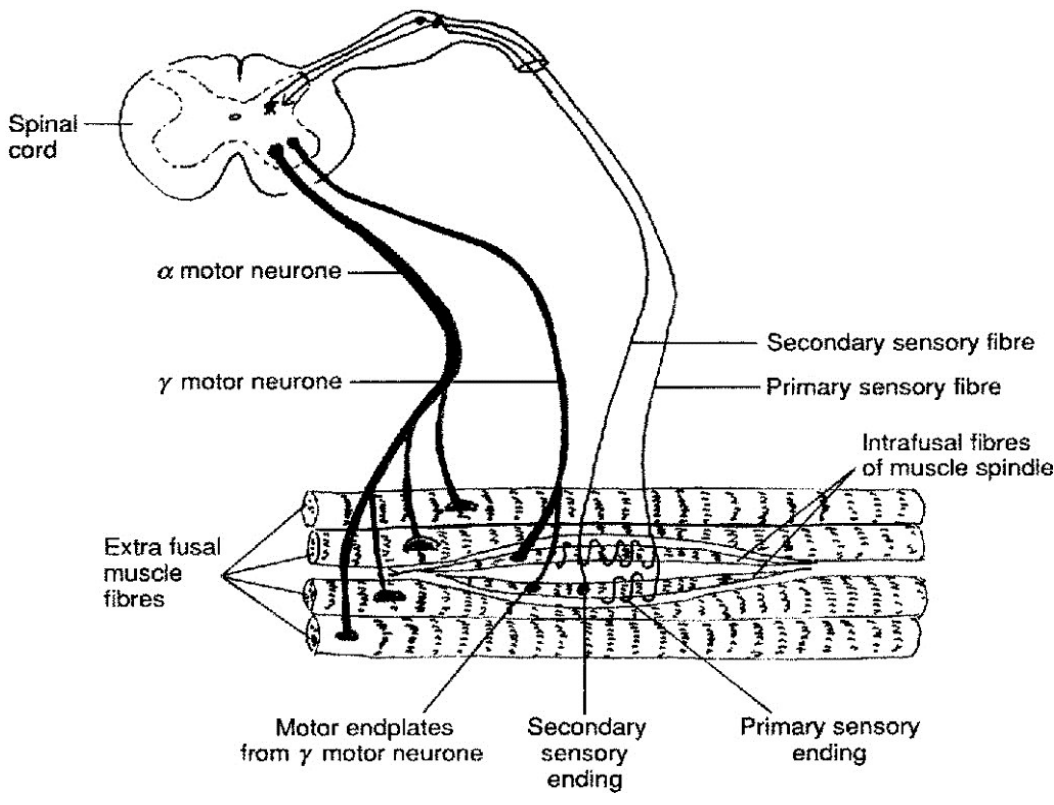

Figure 3: Muscle spindle illustration showing the primary and secondary endings within a muscle. These receptors project electrical impulses to the spinal cord through afferent primary and secondary sensory fibres. Efferent motor neurons that relay information from the spinal cord to the muscle are also shown. Retrieved June 1, 2014 from http://www.answers.com/topic/muscle-spindle. 
areas of premotor cortex associated with the limbs, many neurons respond to tactile stimulation of the corresponding limb and also respond to visual stimuli placed near the tactile receptive field (Graziano, 1999).

\section{Vection}

It has been well established that physical motion can be perceived without actual physical motion stimulating the vestibular and/or the proprioceptive systems, as long as the eyes perceive the changes that would be expected from motion (Burki-Cohen et al., 2007). This illusion of motion is known as vection. While vestibular motion cues immediately yield a sensation of self-motion, large-field (consuming a large degree of the visual field) visual motion can be interpreted as either exocentric motion (where the observer is stationary in a moving environment) or egocentric motion (where the observer is moving through a stationary environment) or a combination of both (Riecke, 2010). When presented with large-field visual motion, the observer typically perceives exocentric motion for up to 30 seconds after motion onset, followed by a brief period of mixed exocentric motion and egocentric motion, and finally exclusive egocentric motion (Dichgans \& Brandt, 1978).

Vection was demonstrated in a series of experiments by Wong and Frost (1978) where, after being presented with rotational optic flow (the pattern of apparent motion of environmental objects, surfaces, and edges in a visual scene caused by the relative motion between an observer and the scene) for 30 seconds, participants reported subjective egocentric motion without any vestibular stimulation. A study by Berthoz, Pavard and Young (1975), explored the weighting of visually induced linearvection (the perception of translational motion) when presented at the same time as forward and backward 
vestibular stimulation. Presenting optic flow to the peripheral of participants' visual field was used to induce linearvection. Latencies to the onset of linearvection were generally reported after one second.

Several other studies within the field of cognitive psychology have uncovered factors that lead to the most effective ways to produce the vection illusion. Dichgans and Brandt (1978) demonstrated that the subjective experience of vection can be enhanced as objects move faster in visual scenes. Furthermore, Brandt, Dichgans and Koenig (1973) showed that subjective circularvection (the perception of rotational motion) velocity increased linearly with increasing stimulus movement up to $120^{\circ}$ per second, and roughly matched the stimulus velocity. Increasing stimulus velocity beyond $120^{\circ}$ per second did not increase circularvection velocity any further. In fact, when stimulus rotation was faster than $120^{\circ}$ per second, the moving stimulus was no longer perceived to be stationary. In terms of simulator applications, this suggests that there might be maximum movement and/or optic flow velocities beyond which simulation effectiveness could weaken, leading to a decrease in psychological fidelity (Riecke, 2010).

Another factor determining the onset and strength of vection is the field of view subtended by the moving visual stimulus. Although Andersen and Braunstein (1985) demonstrated that stimuli as small as $7.5^{\circ}$ can induce vection, larger stimuli generally enhance vection in all measures, and optic flow subtending the full visual field results in the strongest vection to a point where it is indistinguishable from actual egocentric motion (Brandt et al., 1973).

Although earlier studies reported that optic flow presented to the peripheral visual field is more effective in inducing vection than optic flow presented to the central field of 
vision (Johansson, 1977; Brandt et al., 1973), later studies demonstrated that optic flow presented in the peripheral and central fields of vision have similar influences on vection when the visual stimuli are perceived at equal depth. When perceived depth was held constant, vection strength increased linearly with increasing stimulus size, independent of where the stimulus was presented in the visual field (Andersen \& Braunstein, 1985).

With decades of research consistently demonstrating the illusion of egocentric motion using purely visual stimuli, it is quite clear that the visual system has a strong role in the perception of motion (Brandt et al., 1973; Warren \& Kurtz, 1992). Several multisensory studies within aviation research have suggested that motion simulators may produce redundant multisensory cues when presented with visual displays of motion. If the perception of egocentric motion is adequately produced through a visual display, adding physical motion may provide minimal benefits to pilot performance (Kappé, Van Erp \& Korteling, 1999; Eriksson, 2009). The phenomenon of vection is a primary reason why the need for simulating physical motion when using broad angle high-fidelity visual displays is a subject of debate (Burki-Cohen et al., 2007). With the depth of research demonstrating the optimal methods of producing the illusion of egocentric motion and the visual display technology to incorporate these methods, it is possible that motion cues may not be necessary at all for flight simulators. It is, however impossible to ignore the data that consistently demonstrate the more naturalistic behaviours of pilots during simulation training when physical motion cues are provided. This positive behavioural evidence along with pilots' consistent subjective dislike for flight simulators with no motion is what perpetuates the debate over the usefulness of physical motion in simulation-based training. It is now important to further our understanding of exactly 
how the visual system interacts with the vestibular and proprioceptive systems in relation to perceiving physical motion cues through specific types of motion simulators.

\section{Integration of Visual and Motion Cues}

To integrate multisensory information, the nervous system continually changes its emphasis, or weight, on visual, vestibular and proprioceptive information to maintain optimal spatial awareness as environmental conditions change. When combining visual and vestibular/proprioceptive information, the brain does not attend to all unimodal sensory inputs equally. This continuous change in emphasis, or reweighting, requires complex computations that have been demonstrated in many multisensory studies in perception of egocentric motion (Angelaki, Gu \& DeAngelis, 2009, Morgan, DeAngelis \& Angelaki, 2008. The complex reweighting computation underlying visual-vestibular integration can be quantified by an adaptive process in which the central nervous system down-weights (attends less to) unreliable sensory stimuli while simultaneously upweighting (attends more to) more reliable sensory stimuli through top-down processing (e.g., contextual information provided by visual cues), or bottom-up processing (strength of neural activation in unimodal cortical areas) (Blair, Kiemel, Jeka \& Clark, 2012).

It has been well documented that the medial superior temporal cortex (MST) shows the greatest amount of activation to both visual (optic flow) and vestibular (translational head movements in the dark) information related to egocentric motion. The MST has therefore been assumed to be the primary cortical area for the integration of the visual and vestibular senses (Morgan et al., 2008). Recent findings regarding the integration of visual and vestibular stimuli have suggested that visual-vestibular bimodal cells in the MST may not show the same activation patterns as other multisensory areas, 
such as visuotactile and visual-auditory bimodal cells (Angelaki et al., 2009; Morgan et al., 2008). Morgan et al. (2008) explored how the response to visual and vestibular stimuli in the MST related to the responses to the unimodal components presented separately, and whether the strength of both the unimodal stimuli affected the activation pattern. Unlike bimodal cells associated with integrating other sensory information, bimodal cells in the MST predominantly show a subadditive firing pattern (more activity than the greatest unimodal response, but less than the sum of both unimodal responses). A subadditive firing pattern generally suggests that there is a deactivation of unimodal sensory activity after the integration of the multiple senses. Morgan et al. (2008), found that with a combination of visual and vestibular cues, MST cells tended to weight visual information greater than vestibular information. However, when the strength (coherence) of the visual motion cue was reduced, it was found that the MST systematically decreased the visual weights and increased the vestibular weights.

Brandt, Bartenstein, Janek and Dieterich (1998) induced circularvection in human participants using a rotating visual display. Through the use of Positron Emission Tomography (PET) they found significant activation of the medial parieto-occipital visual area (area of the visual cortex close to V5 known for motion perception) as well as deactivation of the posterior insula of the vestibular cortex (area where vestibular and proprioceptive inputs integrate). Lesions in the parieto-occipital visual area have been associated with spatial disorientation due to the perception of tilted vertical orientations (Brandt, Dieterich \& Danek, 1994). Brandt et al. (1998), suggested that this deactivation pattern can also be found in the visual centres associated with the visual-vestibular interaction when the visual stimulus provides weaker stimulation than the vestibular 
stimulus. Seemungal et al. (2013) demonstrated that vestibular activation with a TMS reduced cortical activation in visual area V5, whereas activation in visual areas V1 and V2 remained the same. The effect on V5 activation was vestibular specific, as egocentric motion perception produced by auditory vection had no effect on V5 activation. Theoretically, these activation data allow for a selective suppression of visual egocentric motion perception during physical egocentric motion while leaving other visual functions intact, such as colour and form perception (Seemungal et al., 2013). The visual-vestibular interaction is therefore not necessarily always dominated by either the visual or vestibular/proprioceptive systems but is dependent on the relative strength of the stimuli. If one of the bimodal stimuli is much stronger, then the brain will favour this input modality and a pattern of deactivation will occur in the weaker unimodal sensory centre (Brandt et al., 1998). Mardirossian, Karmali and Merfeld (2014) have, however, demonstrated that when visual and vestibular unimodal inputs are of similar strength, the brain will place greater weight on the visual information.

Mardirossian et al. (2014) found that when estimating the degree of postural sway (full body motion along the bank/tilt axis, in this case produced by standing on a motion platform), the combined visual and vestibular estimate of motion is more accurate than a unimodal vestibular input but is not significantly more accurate than unimodal visual cues alone. Van Beuzekom and Van Gisbergen (2000) presented participants with motion cues at $15^{\circ} /$ second in the full $360^{\circ}$ tilt axis in a dark room, and asked them to estimate their orientation. They found a strong negative correlation of estimate accuracy and tilt magnitude when tilts of $60^{\circ}$ or less were presented, followed by no correlation of estimate accuracy and tilt magnitude between $60^{\circ}$ and $90^{\circ}$, and a positive correlation 
between $90^{\circ}$ and $180^{\circ}$. Van Beuzekom and Van Gisbergen (2000) have shown that the ability to accurately estimate orientation, within the tilt axis, is generally dependent on the magnitude of tilt. This finding may be an important theoretical concept when analyzing the effectiveness of motion cues in a flight simulator. It is possible that benefits of motion cueing may be task specific due to the way in which operators perceive different magnitudes of the motion cueing in simulated tasks. Further research regarding spatial orientation in flight simulators needs to be conducted because misjudgment of spatial orientation is reported to be an important factor in a substantial proportion of aviation accidents. The likelihood of such misjudgment is particularly high when there are conflicting interactions between vestibular and visual input (Regan, 1994).

\section{Present Research}

The goal of the present research was to evaluate how motion cues from a lowfidelity motion-cueing seat affect operators' ability to judge their spatial orientation. Nicholson et al. (2013) found that incongruent cues from a motion seat increased operators' control reversals and overcorrections while correcting from orientation disturbances. These are encouraging results in relation to the way in which operators react to dynamic motion seat cues. The following study extends Nicholson et al.'s work by exploring the effects of dynamic motion seat cueing on an operator's ability to perceive spatial orientation.

Two experiments were conducted to examine how visual and motion seat cues are used to perceive spatial orientation. Experiment 1 explored how adding complementary motion seat cues affected the ability to perceive changes to the orientation of a visual 
display. Experiment 2 investigated how adding complementary visual orientation cues affected the ability to perceive motion cues created by a dynamic motion seat.

\section{Experiment 1}

Experiment 1 was designed to determine if adding consistent motion cues from a dynamic motion seat would enhance the ability to accurately estimate the magnitude of bank angles presented on a visual display (i.e., attitude indicator). Inconsistent motion seat cues were also presented in order to explore whether conflicting motion seat cues would interfere with the ability to estimate bank angles presented on an attitude indicator. It is hypothesized that when visual and motion seat cues are inconsistent, accuracy in estimating inconsistency will increase with larger magnitude disparities. It is expected that consistent motion seat cues will not significantly enhance one's ability to estimate the magnitude of motion within a visual display beyond no motion. Given the results of Nicholson et al. (2013) and Mardirossian et al. (2014) inconsistent motion seat cues are expected to significantly decrease an individual's ability to estimate the magnitude of visual motion cues.

\section{Method}

\section{Participants}

Twenty-six participants (19 females) were recruited from the Carleton University psychology participant pool and were compensated with $1 \%$ course credit. Ages ranged from 18 to 33 years $(M=21.54, S D=3.13)$. All participants were required to have normal or corrected-to-normal vision and not to have any current or past problems with vertigo or back injuries.

\section{Design}


A 3 (Motion Condition: No Motion vs. Consistent Motion vs. Inconsistent Motion) x 4 (Visual Bank Angle: $10^{\circ}$ vs. $20^{\circ}$ vs. $30^{\circ}$ vs. $40^{\circ}$ ) repeated measures design was used. Both factors were mixed and trials from these conditions were randomly presented such that there were an equal number of trials from each motion condition and each visual bank angle.

\section{Apparatus and Materials}

An ACME America VMax True Q Dynamic Motion Seat provided motion cues that simulated helicopter roll at bank angles of $10^{\circ}, 20^{\circ}, 30^{\circ}$, or $40^{\circ}$. The physical "tilt" of the motion seat cues was drastically less than these bank angles, but was subjectively representative of a helicopter rolling at these magnitudes. Each motion cue consisted of one movement, either left or right that was generated by two separate parts of the motion seat that moved simultaneously. The first part was the back pan, which swayed left/right for up to approximately $500 \mathrm{~ms}$ depending on the bank angle. The second part was the seat pan, which tilted left/right based on the magnitude of the bank angle (see Figure 4). Once the back pan and seat pan movements reached the desired bank angle, the seat slowly returned to its resting state $\left(0^{\circ}\right.$ bank angle) such that any movement following the execution of motion cue was not obviously apparent. Participants were secured to the motion seat by lap and shoulder belts with their feet resting flat on a level surface. 


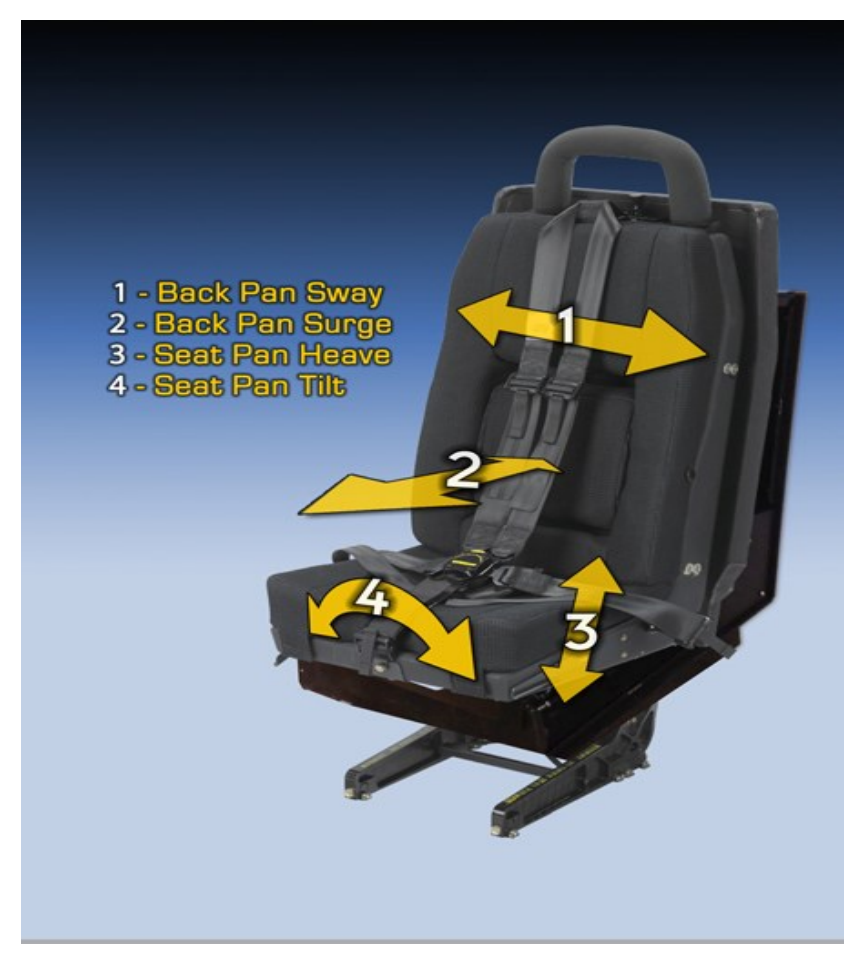

Figure 4. Dynamic motion seat used in Experiments 1 and 2. This figure illustrates the four possible types of motion cues that the seat can provide, however only simultaneous back pan sway (1) and seat pan tilt (4) motion cues were used here. Retrieved June 1, 2014 from http://www.acme-worldwide.com/dynamic_motion_seat_VMax.htm.

Visual motion cues were presented on an inside-out attitude indicator ${ }^{1}$ (see Figure 5), where a fixed aircraft symbol was presented on a horizon line. The horizon tilted at $10^{\circ}, 20^{\circ}, 30^{\circ}$, or $40^{\circ}$, left/right to indicate the magnitude and direction of the bank angle and then returned to level ( $0^{\circ}$ bank angle) once the desired bank angle was reached. The visual motion cues displayed on the attitude indicator and the physical motion cues provided by the motion seat occurred simultaneously. Because the motion seat cues did not physically move to the desired bank angle while the visual motion cues did, the visual cues had a faster roll rate than the motion seat cues in order to reach the desired bank

\footnotetext{
${ }^{1}$ As opposed to an outside-in attitude indicator, where the horizon line remains fixed and the aircraft symbol tilts to display the aircraft's orientation.
} 
angle at the same time. The attitude indicator subtended a visual angle of approximately $11^{\circ}$ and was presented against a black background on a 47-inch LCD monitor with a 60 $\mathrm{Hz}$ refresh rate. The monitor was centred approximately $127 \mathrm{~cm}$ in front of the participants and remained stationary throughout the procedure. A PC

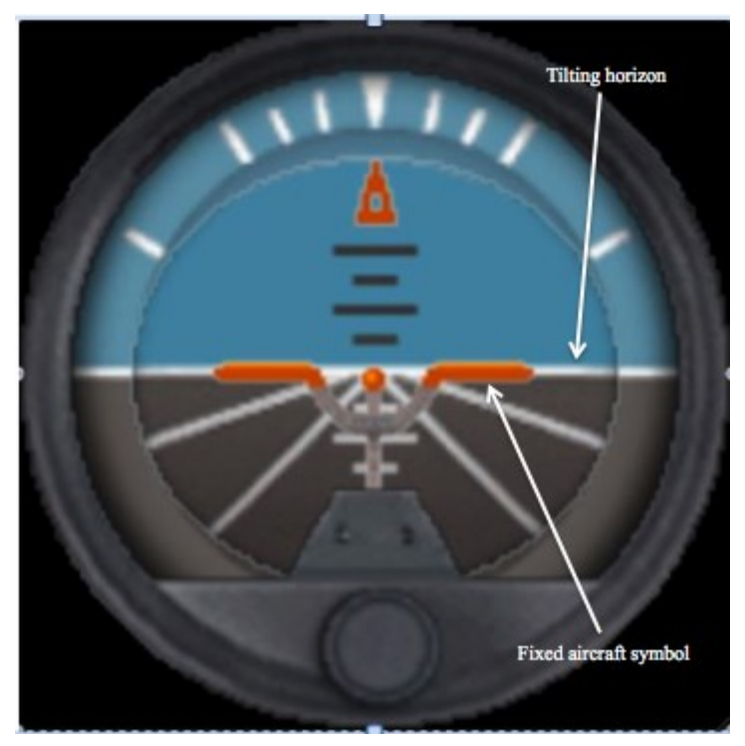

Figure 5. Inside-out attitude indicator used to provide visual motion cues in Experiments 1 and 2. The white horizon line and background tilts at varying angles while the aircraft symbol remains fixed.

connected to the monitor and the motion seat was responsible for synchronizing the visual and physical motion cues.

Participants responded on a Cedrus RB-740 button box (see Figure 6). Participants first pressed either a "Y" or "N" button to indicate if the motion seat and visual cues matched or did not match and then pressed either a " 10 " " 20 " " 30 " or " 40 " button to indicate the magnitude of the visual cue. 


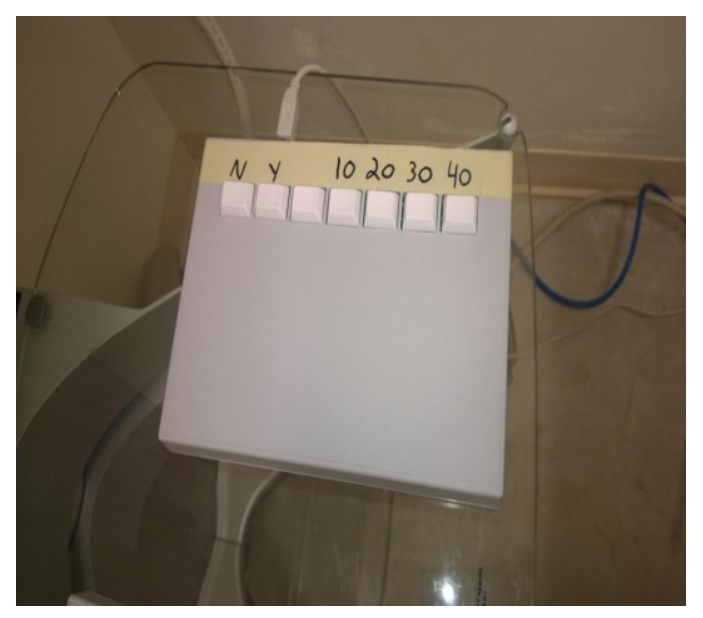

Figure 6. Button box used in Experiments 1 and 2. Participants first pressed either "Y" or "N" to indicate if the physical and visual cues matched or did not match and then pressed either " 10 " "20" " 30 " or " 40 " to indicate the magnitude of the visual cue (Experiment 1) or the motion seat cue (Experiment 2).

\section{Procedure}

Participants were tested individually in a single session in a noise-controlled lab. Participants provided informed consent and demographic information, and were then seated and secured in the motion seat.

Participants began with a familiarization block, where they were trained to distinguish between physical (motion seat) and visual (attitude indicator) bank angle magnitudes $\left(10^{\circ}, 20^{\circ}, 30^{\circ}\right.$ or $\left.40^{\circ}\right)$. The familiarization block consisted of 16 motion cues (two per bank angle in each direction) accompanied by a consistent visual cue (e.g., a $40^{\circ}$ tilt to the right with the attitude indicator moving $40^{\circ}$ to the right). The familiarization block always began with the $40^{\circ}$ physical/visual cues and proceeded in descending order of bank angle magnitude.

Once the familiarization block was completed, participants were instructed to first indicate whether the magnitudes of the physical motion cue provided by the motion seat 
and the visual cue shown on the attitude indicator were consistent or inconsistent ${ }^{2}$. Then, they were to estimate the bank angle of the visual motion cue, as quickly and accurately as possible. Participants completed one practice block of 15 trials (five per each motion condition) and then four experimental blocks with 36 trials each, for a total of 144 experimental trials. In the no motion condition, a visual cue was presented without a physical cue (i.e., the motion seat did not move). In the consistent motion condition, the magnitude and direction of visual and motion cues were identical. In the inconsistent motion condition, the visual and motion cues were in the same direction but differed in terms of magnitude. Participants were debriefed following the experimental trials and any outstanding questions were answered. The entire experiment was approximately 40 minutes.

\section{Results and Discussion}

It was not possible to synchronize the motion seat with the button box, therefore reaction times in the consistency judgment and bank angle estimation tasks were not analyzed. Post-hoc comparisons used 95\% confidence intervals (Jarmasz \& Hollands, 2009).

Consistency Judgment Data. Consistency judgments were not recorded in the no motion condition, thus these data were analyzed using a 2 (Motion Condition: consistent motion vs. inconsistent motion) x 4 (Visual Bank Angle: $10^{\circ}$ vs. $20^{\circ}$ vs. $30^{\circ}$ vs. $40^{\circ}$ ) repeated measures analysis of variance (ANOVA) (see Figure 7). There was a significant main effect of visual bank angle, $F(3,75)=23.06, \mathrm{MSE}=.02, p<.001, \eta p^{2}=.50$.

\footnotetext{
${ }^{2}$ Accuracy in determining whether each motion seat cue matched the visual motion cue was included as a secondary analysis. Participants were asked to complete this task primarily to force them to attend to both the visual and motion seat cues simultaneously.
} 
Participants were significantly more accurate in judging consistency when the visual cue was $10^{\circ}(M=86.5 \%, S E=2.4)$, than when it was $20^{\circ}(M=64.1 \%, S E=2.3), 30^{\circ}(M=$ $66.7 \%, S E=2.5)$, or $40^{\circ}(M=71.7 \%, S E=2.0)$. There were no other significant differences in consistency judgments between visual bank angles. The main effect of

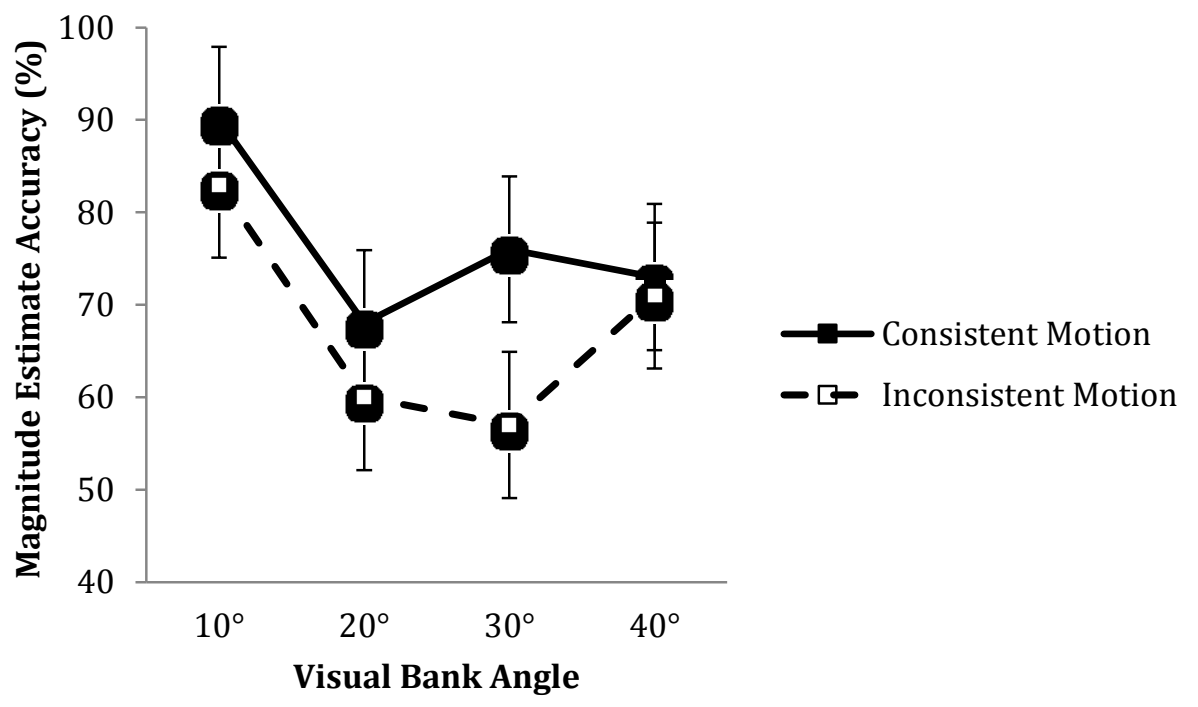

Figure 7. Consistency judgment accuracy as a function of visual bank angle and motion condition.

motion condition was also significant, $F(1,25)=7.16, \mathrm{MSE}=.06, p=.01, \eta p^{2}=.24$, with higher accuracy in the consistent motion condition $(\mathrm{M}=76.8 \%, \mathrm{SE}=0.2)$ than in the inconsistent condition $(\mathrm{M}=67.7 \%, \mathrm{SE}=0.2)$. There was no Motion Condition $\mathrm{x}$ Visual Bank Angle interaction, $F(3,69)=2.21, \mathrm{MSE}=.03, p=.10, \eta p^{2}=.09$.

Accuracy in the inconsistent motion condition was analyzed using the magnitude of the disparity between the motion and visual cues as a factor. This analysis was used to explore whether the size of the cue disparity had an effect on accuracy. A one-way repeated measures ANOVA with three levels (Disparity: $10^{\circ}, 20^{\circ}, 30^{\circ}$ ) showed a significant main effect of disparity between the motion and visual cues, $F(2,50)=$ 
175.50, MSE $=.008, p<.001, \eta p^{2}=.88$. Participants were significantly more accurate in estimating consistency when the disparity magnitude was $30^{\circ}(M=95.1 \%, S E=1.9)$, than when it was $20^{\circ}(M=78.8 \%, S E=2.5)$, and $10^{\circ}(M=46.4 \%, S E=3.1)$, and significantly more accurate when it was $20^{\circ}$ than $10^{\circ}$ (see Figure 8 ).

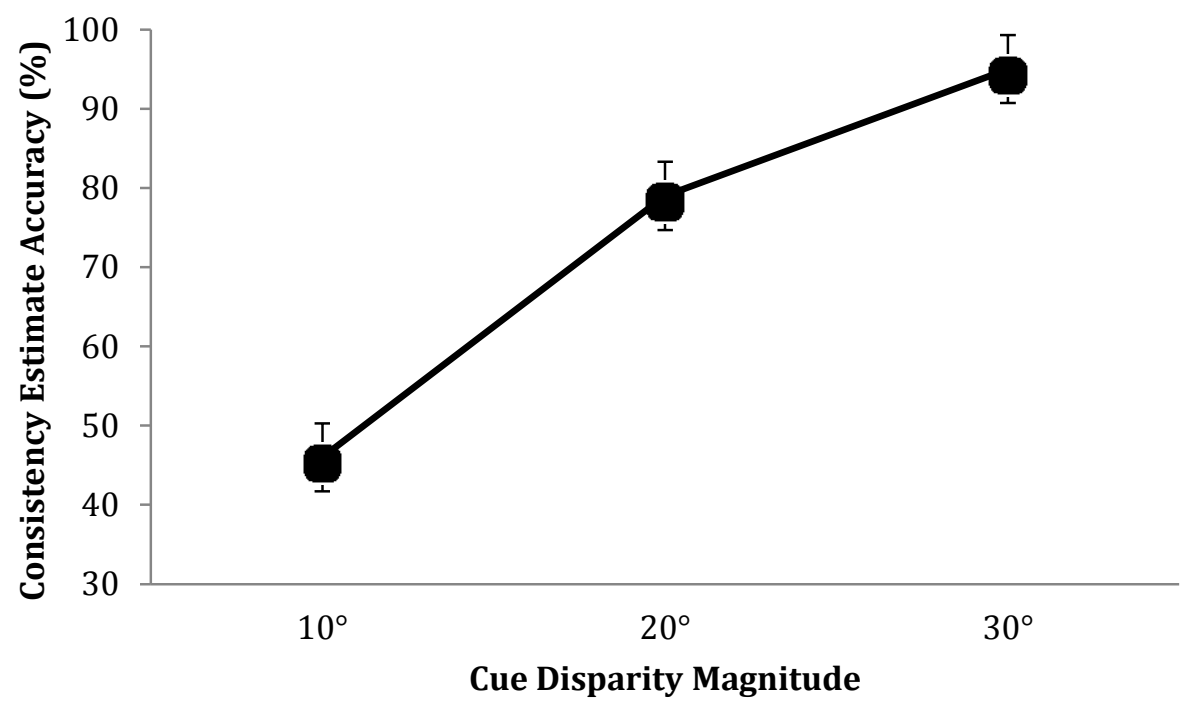

Figure 8. Consistency judgment accuracy as a function of visual and motion-cue disparity (Experiment 1).

Bank Angle Estimation Data. Bank angle estimate data were analyzed using a 3 (Motion Condition: no motion vs. consistent motion vs. inconsistent motion) x 4 (Visual Bank Angle: $10^{\circ}$ vs. $20^{\circ}$ vs. $30^{\circ}$ vs. $40^{\circ}$ ) repeated measures ANOVA (see Figure 9). There was a significant main effect of visual bank angle, $F(3,75)=8.32, \mathrm{MSE}=.09, p=$ $.001, \eta p^{2}=$ 


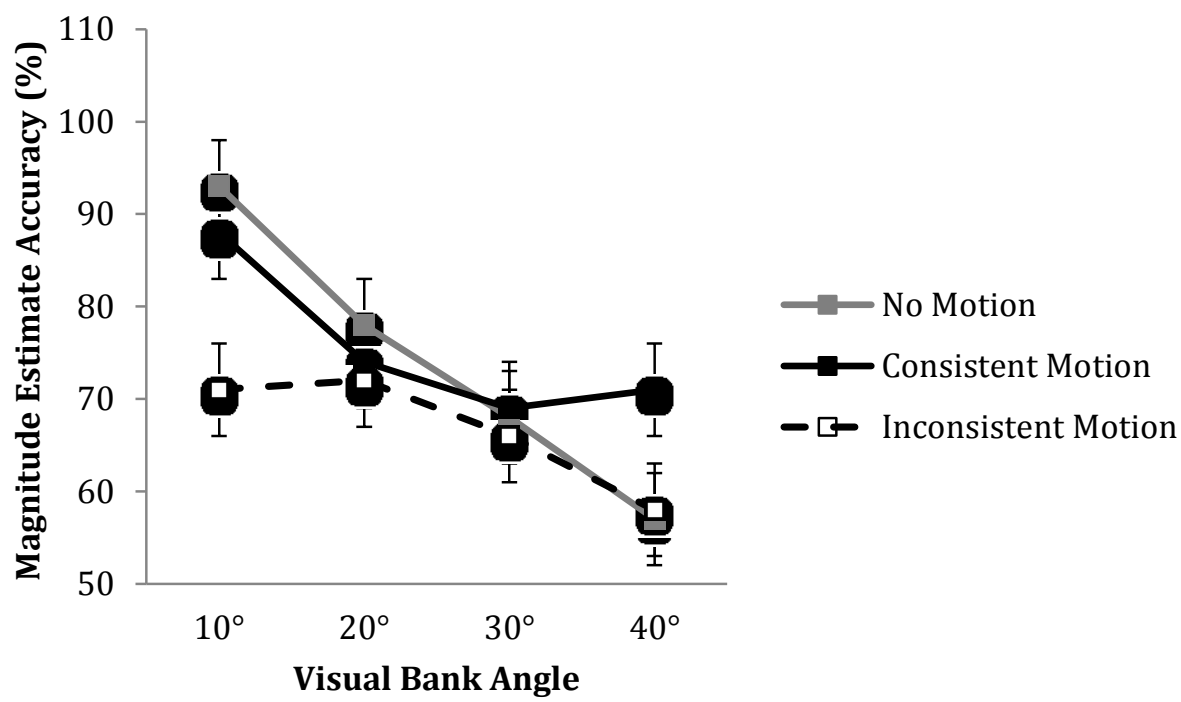

Figure 9. Bank angle estimation accuracy as a function of visual bank angle and motion condition.

.25. where participants were more accurate in estimating bank angles when the visual cue was $10^{\circ}(M=84.3 \%, S E=3.0)$, than when it was $20^{\circ}(M=74.5 \%, S E=4.3), 30^{\circ}(M=$ $67.5 \%, S E=4.1)$, or $40^{\circ}(M=62.3 \%, S E=3.4)$. Accuracy was also significantly greater at $20^{\circ}$ than at $30^{\circ}$ or $40^{\circ}$.

The main effect of motion condition was also significant, $F(2,50)=11.51$, MSE $=.02, p<.001, \eta p^{2}=.32$. Accuracy was significantly greater in the no motion $(M=$ $74.1 \%, S E=2.7)$ and consistent motion conditions $(M=75.6 \%, S E=2.5)$, than in the inconsistent motion condition $(M=66.8 \%, S E=2.8)$. There was a significant Motion Condition $\mathrm{x}$ Visual Bank Angle interaction, $F(6,150)=6.57, p<.001, \eta p^{2}=.21$. An examination of Figure 8 reveals that the interaction was primarily driven by the motion condition (consistent vs. inconsistent) having large effects at the two extremes of the visual bank angle condition $\left(10^{\circ}\right.$ and $\left.40^{\circ}\right)$, with relatively little effect at $20^{\circ}$ or $30^{\circ}$. That is, relative to the no motion condition, inconsistent motion cues decreased bank angle 
estimate accuracy at $10^{\circ}$ but did not appear to affect estimation accuracy at $20^{\circ}, 30^{\circ}$ or $40^{\circ}$. Relative to the no motion condition, consistent motion cues significantly increased accuracy only at $40^{\circ}$ but not at $10^{\circ}, 20^{\circ}$ or $30^{\circ}$.

Accuracy in the inconsistent motion condition was analyzed using the magnitude of the disparity between the motion and visual cues as a factor. This analysis was used to explore whether the size of the cue disparity had an effect on accuracy. A one-way repeated measures ANOVA with three levels (Disparity: $10^{\circ}, 20^{\circ}, 30^{\circ}$ ) showed that bank angle estimation accuracy did not differ as a function of the disparity between the motion and visual cues, $F(2,50)=2.95, \mathrm{MSE}=.02, p=.08, \eta p^{2}=.11$.

The present study found a significant main effect of visual bank angle on accuracy. Participants could more accurately judge consistency at $10^{\circ}$ compared to $20^{\circ}$, $30^{\circ}$ and $40^{\circ}$. Motion cue consistency also had a significant main effect as participants were significantly more accurate when the cues were consistent than when the cues were inconsistent. Participants' ability to judge cue consistency was significantly more accurate at greater magnitude disparities (e.g., $30^{\circ}$ ) than lower disparities (e.g., $\left.10^{\circ}\right)$. This finding supports the hypothesis that when visual and motion seat cues are inconsistent, accuracy will increase with larger magnitude disparities. This finding shows that participants could accurately perceive the difference between high magnitudes and low magnitudes of visual motion, or the inverse, and became less accurate at smaller disparities.

Consistent motion seat cues at lower magnitudes did not significantly enhance participants accuracy over no motion. Indeed, consistent motion only provided a significant benefit over no motion (and inconsistent motion) at $40^{\circ}$ bank angles. Although 
there was a significant main effect of visual bank angle magnitude, these findings cannot directly be compared with Van Beuzekom et al.'s (2000) results, as participants in this study were estimating bank angles based on the information provided by the attitude indicator whereas Van Beuzekom et al. required participants to estimate the magnitude of physical motion. When collapsed across motion conditions, bank angle estimate accuracy showed the same negative relationship with magnitude as Van Beuzekom et al. (2000), in that lower bank angle magnitudes produced higher estimate accuracy.

The finding that there was no difference in bank angle estimation accuracy between the consistent motion condition and the no motion condition at lower bank angles is consistent with Nicholson et al. (2013) and Mardirossian et al. (2014). The finding that a consistent motion cue does not help an observer estimate the magnitude of a visual cue may reflect a greater weighting of the visual cue when it occurs simultaneously with a motion cue. Given the characteristics of the task used here, it may be the case that the motion cue is providing a redundant and less discernible cue than that provided by the visual information and is therefore being down-weighted. The finding that inconsistent motion cues interfere with the estimation of a visual bank angle mirrors the findings reported by Nicholson et al. (2013) and Mardirossian et al. (2014). This interference effect suggests that although some vestibular information from the motion cue is down-weighted (or deactivated) during the integration the visual and vestibular systems, this vestibular information is still processed and subsequently interferes with the processing of inconsistent spatial orientation information. 


\section{EXPERIMENT 2}

The purpose of Experiment 2 was to explore whether consistent and inconsistent visual cues would affect the perception of vestibular motion in the same way that consistent and inconsistent vestibular motion affected the perception of visually induced motion in Experiment 1. To this end, participants were to estimate the magnitude of a bank angle based on cues provided by a motion seat while viewing either consistent or inconsistent visual cues displayed on an attitude indicator. Given that the participant's task is to estimate the bank angle provided by the motion seat, Experiment 2 is more comparable to Van Beuzekom et al. (2000) than Experiment 1. Based on Van Beuzekom et al.'s findings, it is hypothesized that consistency judgments and bank angle estimates will be perceived more accurately at lower bank angles than at higher bank angles. It is also expected that, relative to baseline (no visual cues), consistent visual cues will significantly enhance the ability to estimate the magnitude of the physical cues provided by the motion seat and inconsistent visual motion cues will significantly impair this ability. This hypothesized pattern of results was demonstrated by Mardirossian et al. (2014) using a postural sway paradigm.

Experiment 2 incorporates the same materials and methods as Experiment 1, except that participants were asked to estimate the magnitude of the bank angle based on the physical cues provided by the motion seat and not the visual cues displayed on the attitude indicator. In the no visual condition, the attitude indicator was not displayed during a physical motion seat cue. In the consistent visual condition, the attitude indicator moved in the same direction and magnitude as the cue provided by the motion seat. In the 
inconsistent visual condition, the attitude indicator moved in the same direction as the cue provided by the motion seat, but at a different bank angle magnitude.

\section{Method}

\section{Participants}

Thirty participants (17 females) were recruited from the Carleton University community and psychology participant pool and were compensated with $1 \%$ course credit. Ages ranged from 18 to 27 years $(M=20.4, S D=2.7)$, all participants were required to have normal or corrected-to-normal vision and not to have any current or past problems with vertigo, motion sickness, or back injuries.

\section{Design}

A 3 (Visual Condition: No Visual vs. Consistent Visual vs. Inconsistent Visual) x 4 (Motion Bank Angle: $10^{\circ}$ vs. $20^{\circ}$ vs. $30^{\circ}$ vs. $40^{\circ}$ ) repeated measures design was used. Both factors were mixed and trials from these conditions were randomly presented such that there were an equal number of trials from each motion condition and each visual bank angle.

\section{Procedure}

Experiment 2 was identical to Experiment 1 except that the participant's task was to estimate the magnitude of the bank angle based on the physical cues provided by the motion seat instead of the visual cues displayed on the attitude indicator.

\section{Results and Discussion}

It was not possible to synchronize the motion seat with the button box, therefore reaction time data were not analyzed. Post-hoc comparisons used 95\% confidence intervals (Jarmasz \& Hollands, 2009) 
Consistency Judgment Data. Consistency judgments were not recorded in the no visual condition, thus these data were analyzed using a 2 (Visual Condition: consistent visual vs. inconsistent visual) x 4 (Motion Seat Bank Angle: $10^{\circ}$ vs. $20^{\circ}$ vs. $30^{\circ}$ vs. $40^{\circ}$ ) repeated measures ANOVA. As shown in Figure 10, there was a significant main effect of motion seat bank angle magnitude, $F(3,87)=56.43, \mathrm{MSE}=.01, p<.001, \eta p^{2}=.66$. Participants were significantly more accurate in judging consistency when the motion seat cue was $10^{\circ}(M=88.8 \%, S E=2.0)$, than when it was $20^{\circ}(M=60.7 \%, S E=2.2)$, $30^{\circ}(M=71.5 \%, S E=1.5)$, or $40^{\circ}(M=75.9 \%, S E=1.9)$.

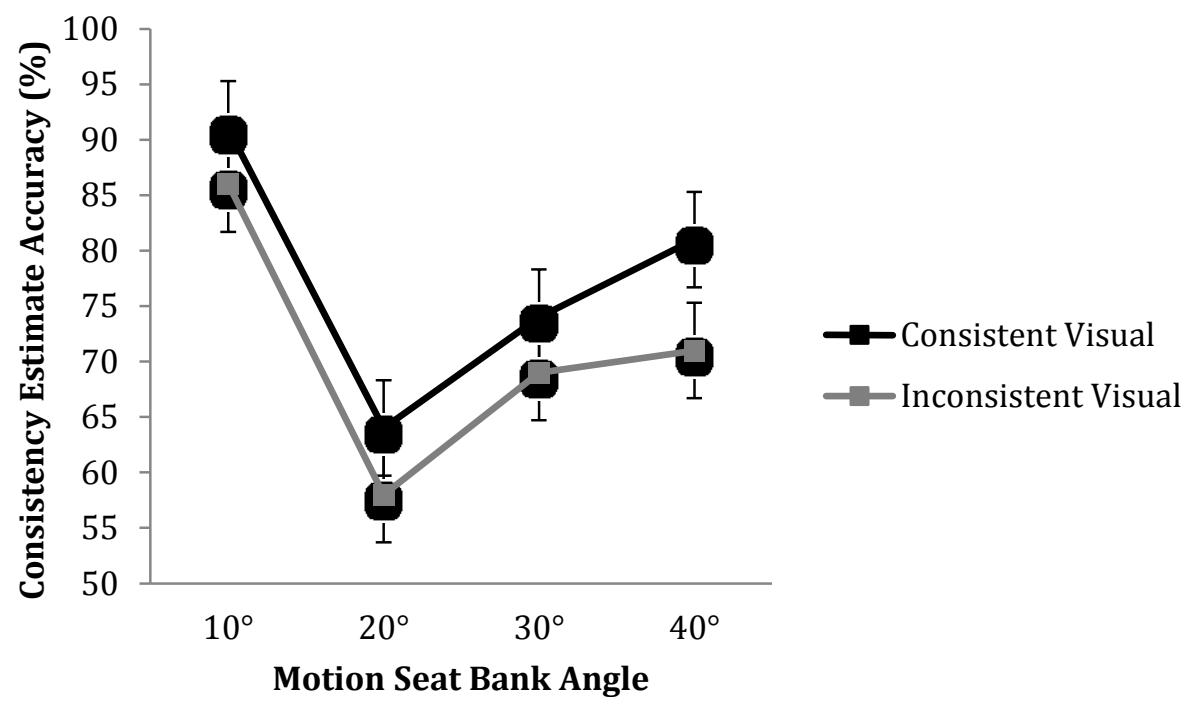

Figure 10. Consistency estimation accuracy as a function of motion seat bank angle and visual condition.

Estimating consistency was also more accurate at $40^{\circ}$ than it was at $20^{\circ}$ or $30^{\circ}$ bank and more accurate at $30^{\circ}$ than $20^{\circ}$ bank angles. Although consistency judgment accuracy was higher in the consistent visual condition $(\mathrm{M}=77.5 \%, \mathrm{SE}=.02)$ than in the inconsistent condition $(\mathrm{M}=70.9 \%, \mathrm{SE}=.03)$, this difference did not reach significance, $F(1,29)=$ 
2.83, MSE $=.09, p=.10, \eta p^{2}=.09$. There was no Visual Condition $\mathrm{x}$ Motion Seat Bank Angle interaction, $F(3,87)=.79, \mathrm{MSE}=.01, p=.50, \eta p^{2}=.03$.

Consistency judgment accuracy in the inconsistent visual condition was analyzed using the magnitude of the disparity between the motion and visual cues as a factor. This analysis was used to explore whether the size of the cue disparity had an effect on consistency judgment accuracy. A one-way repeated measures ANOVA with three levels (Disparity: $10^{\circ}$ vs. $20^{\circ}$ vs. $30^{\circ}$ ) showed a significant main effect of disparity, $F(2,58)=$ 175.97, MSE $=.007, p<.001, \eta p^{2}=.86$. Pairwise comparisons using $95 \%$ confidence revealed that participants were significantly more accurate in estimating consistency when the disparity magnitude was $30^{\circ}(M=97.1 \%, S E=0.9)$, than when it was $20^{\circ}(M=$ $83.1 \%, S E=2.4)$, or $10^{\circ}(M=55.5 \%, S E=2.2)$, and significantly more accurate when it was $20^{\circ}$ than $10^{\circ}$ (see Figure 11).

Bank Angle Estimate Data. Bank angle estimate data were analyzed using a 3 (Visual Condition: no visual vs. consistent visual vs. inconsistent visual) x 4 (Motion Seat Bank Angle: $10^{\circ}$,vs. $20^{\circ}$ vs. $30^{\circ}$ vs. $40^{\circ}$ ) repeated measures ANOVA. As shown in Figure 12, there was a significant main effect of motion seat bank angle $F(3,87)=$ $51.58, p<.001, \eta p^{2}=.64$. Participants were significantly more accurate in estimating bank angles when the motion seat cue was $10^{\circ}(M=87.9 \%, S E=2.0)$, than when it was $20^{\circ}(M=50.4 \%, S E=3.0), 30^{\circ}(M=53.5 \%, S E=2.0)$, or $40^{\circ}(M=55.6 \%, S E=3.0)$. There were no other significant differences in bank angle estimates between motion seat bank angles. 


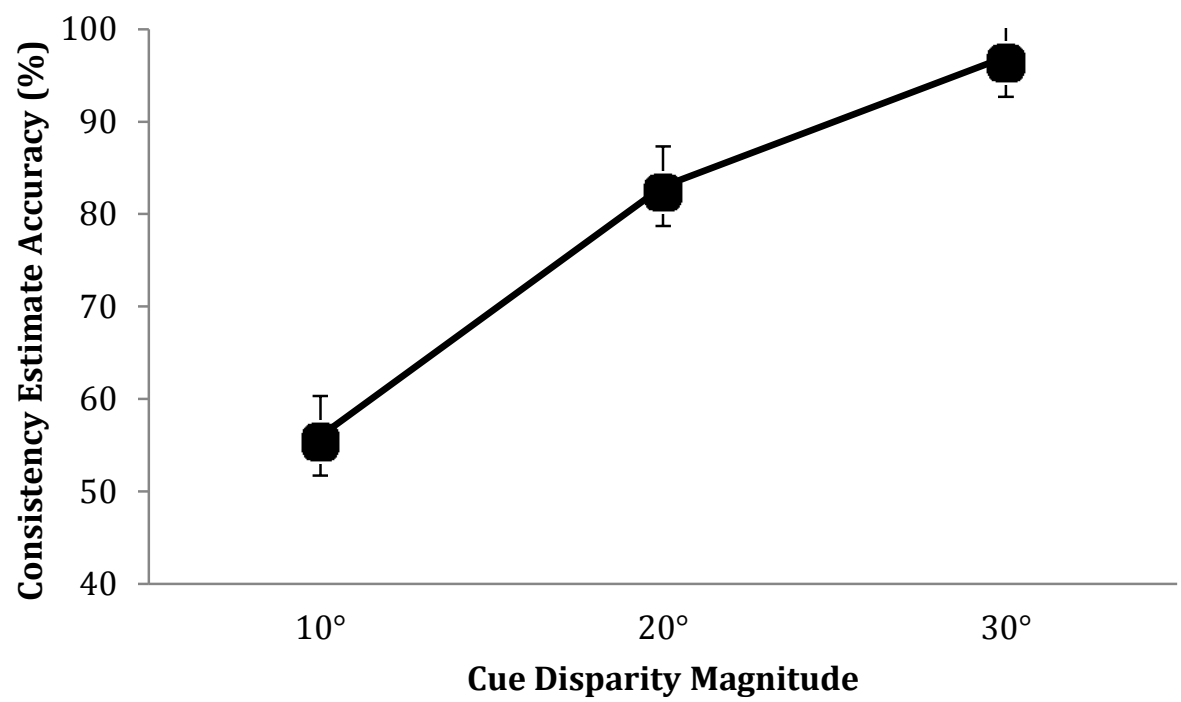

Figure 11. Consistency judgment accuracy as a function of visual and motion-cue disparity (Experiment 2).

The main effect of visual condition was also significant, $F(2,58)=67.35, \mathrm{MSE}=$ $.03, p<.001, \eta p^{2}=.70$. Motion seat bank angle estimation accuracy was significantly greater in the no visual $(M=63.9 \%, S E=1.7)$ and consistent visual conditions $(M=$ $70.4 \%, S E=2.0)$, than in the inconsistent motion condition $(M=50.5 \%, S E=1.9)$. There was a significant Visual Condition x Motion Seat Bank Angle interaction, $F(6,174)=$ $6.62, p<.001, \eta p^{2}=.19$. Inconsistent visual cues significantly decreased estimate accuracy at $10^{\circ}, 20^{\circ}$, and $40^{\circ}$ magnitude, below no visual, while consistent visual cues only improved accuracy at $40^{\circ}$ magnitude above no motion. 


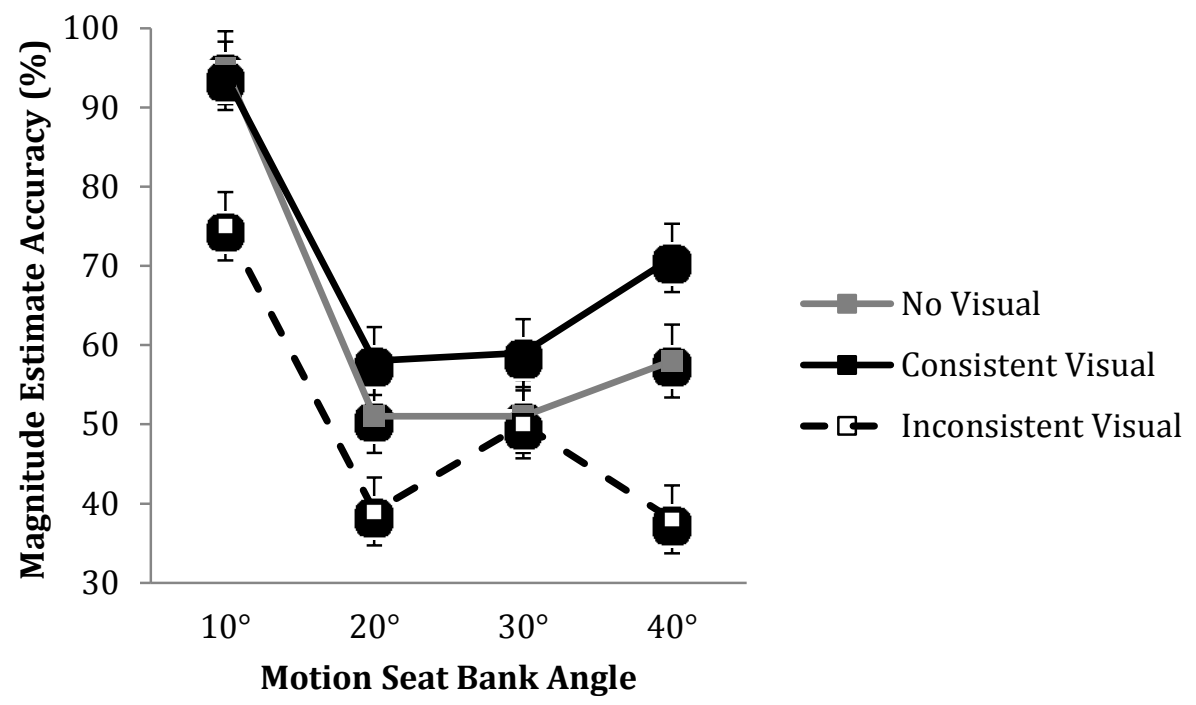

Figure 12. Bank angle estimation accuracy as a function of motion seat bank angle and visual condition.

Bank angle estimate accuracy in the inconsistent motion condition was analyzed using the magnitude of the disparity between the motion and visual cues as a factor. This analysis was used to explore whether the size of the cue disparity had an effect on bank angle estimate accuracy. A one-way repeated measures ANOVA with three levels (Disparity: $10^{\circ}$ vs. $20^{\circ}$ vs. $30^{\circ}$ ) showed that bank angle estimation accuracy did not differ as a function of the disparity between the motion and visual cues, $F(2,58)=3.06$, MSE $=$ $.01, p=.07, \eta p^{2}=.10$.

The main effect of motion seat bank angle magnitude in the present study is similar to Van Beuzekom et al.'s (2000) results in that the lowest bank angle condition $\left(10^{\circ}\right)$ yielded the highest magnitude estimation accuracy. However, unlike Van Beuzekom et al., bank angle estimation accuracy did not decline as the motion cues progressively increased beyond $20^{\circ}$. In the present study estimate accuracy significantly decreased beyond $10^{\circ}$ and then statistically leveled off. This discrepancy might be 
attributable to differences in the motion cue velocities and accelerative forces produced by the different motion systems. Van Beuzekom et al., (2000) presented physical motion cues at a roll rate of $15 \%$ second compared to $200 \%$ second in the present study.

The main effect of motion condition is consistent with Mardirossian et al.'s (2014) findings. Specifically, the presence of an inconsistent visual cue impaired performance on this task at all magnitudes with the exception of $30^{\circ}$ in the present study. A consistent visual cue enhanced the ability to estimate bank angle magnitude using a physical motion cue, although this only occurred at $40^{\circ}$ bank angles. Consistent visual motion cues may not have provided the same benefits in Experiment 2 as it did in Mardirossian et al.'s (2014) study due to the restricted bank angle magnitudes used here.

\section{General Discussion}

The present study explored the effects of dynamic motion seat cueing on operators' perceived spatial orientation, by examining the accuracy with which operators could perceive their spatial orientation across varying magnitudes of bank and varying conditions of cue consistency. Participants were presented with simultaneous displays of visual motion (attitude indicator) and dynamic motion seat cues, and were required to estimate bank angles based on either the visual motion cues provided on an attitude indicator (Experiment 1) or the physical cues provided by a motion seat (Experiment 2).

Both experiments provided evidence to support Van Beuzekom et al. (2000), in that bank/tilt motion is processed differently depending on bank angle magnitude, with smaller magnitudes being easier to estimate. Unlike Van Beuzekom et al. (2000), the present study did not investigate the full range of bank as the dynamic motion seat was on a fixed base platform and was only capable of providing a limited range of bank motion. 
The results of Experiment 1 are similar to many previous motion cueing studies (e.g., Burki-Cohen et al., 2007; Hall, 1989). Providing complementary motion cues through the use of a dynamic motion seat did not provide any benefits beyond a visual display. With very little evidence supporting a benefit of full platform motion, and similar results using alternative motion cueing, it may be the case that motion cueing does not provide significant benefits to tasks that primarily involve vision. Experiment 2 provided evidence that estimating bank angles based on physical (motion) cues was assisted by the inclusion of consistent and concurrent multisensory stimuli (i.e., visual cues), as opposed to motion cues alone. It has been shown in human (Brandt et al., 1973) and primate (Morgan et al., 2008) studies that the brain selectively deactivates one of the visual or vestibular systems during their integration but generally favours visual information. Therefore, redundant vestibular cues do not tend to enhance this specific multisensory perception. Furthermore, the present study uses a simple, 2-dimensional inside-out attitude indicator to represent visual motion. If inconsistent visual information from the attitude indicator produces significant decreases in the accuracy of vestibular motion estimates, it may be that the visual system is so dominant in the visual-vestibular interaction, that circularvection or linearvection need not be presented in order for the brain to weight visual motion information greater than vestibular motion information. Experiment 1 shows that during the integration of the visual and vestibular systems, the MST does not completely deactivate the lesser-weighted sense (here, activation in the vestibular system). If the lesser-weighted sense was completely deactivated, then the inconsistent motion condition would not have impaired bank angle 
estimation accuracy. This significant decrease in accuracy demonstrates that there was some form of interference from the vestibular system while perceiving visual motion.

\section{Future Directions}

Lee (1989) suggested that instead of using high-fidelity motion systems, it might be more economical to implement motion seats for cueing the onset of motion, as opposed to simulating the entire movement of the aircraft. This idea has been shown to be successful when simulating surge (Burki-Cohen, 2007). Experiment 1, however, shows that even if the motion-seat cue is congruent with the visual display, the inclusion of motion cues that are discrepant with the visual display may compromise the operator's sense of spatial orientation.

Despite inconsistent evidence, pilots and the FAA strongly support the inclusion of motion cueing systems in training. Subjectively, pilots feel that motion cueing adds to the psychological fidelity of the simulation and have shown a consistent dislike for simulators that do not provide motion cues. As noted by Miller (1953), capturing the most naturalistic behaviours possible within a simulation is extremely important. Because there is little evidence that high-fidelity motion systems provide significant benefits over lower-fidelity motion systems (e.g., dynamic motion seats), it would be logical to continue researching the utility of dynamic motion seats in aviation training. While dynamic motion seats do not provide a benefit over platform motion in terms of operator performance and transfer of training, the financial burden of procuring, operating and maintaining a motion seat is far less than it is for a full motion platform.

Although dynamic motion seats have shown few benefits of consistent motion cueing, the present study and Nicholson et al.'s (2013) work have demonstrated that 
inconsistent and incongruent motion cues generally disrupt performance in recovering from unusual orientations. Because pilots are usually trained to ignore vestibular cues during flight and focus completely on their instruments, using a dynamic motion seat to train pilots to ignore incongruent motion cues may be an effective approach to training.

\section{Limitations}

A limitation of the present study was that reaction times to visual and motion seat cues were not accurately measured. The software provided by the motion seat manufacturer did not allow for it to be synchronized with the button box. As such, reaction times were recorded after $200 \mathrm{~ms}$ of motion cue onset, regardless of the bank angle, despite slight temporal differences between magnitudes.

\section{Conclusion}

In conclusion, including motion cueing in flight simulators is expensive and thus centralizes training to the few institutions that can afford this initial and long-term financial and technical investment. Dynamic motion seats provide a low-cost alternative to high fidelity motion systems and have been shown to elicit the same pilot performance within a limited number of flight tasks. The present study examined the ability of operators to perceive their spatial orientation in a dynamic motion seat through the use of both visual and motion seat cues. In Experiment 1 it was found that adding complementary motion seat cues did not benefit participants' ability to perceive their orientation through a visual display, although motion cues that were inconsistent with those indicated on the visual display disrupted orientation perception. In Experiment 2, it was found that adding complementary visual motion cues did benefit participants' ability to perceive their orientation during motion seat cues. These findings show that motion 
cues for dynamic motion seats affect operators' perception of spatial orientation. It is likely that these results were observed under these testing conditions due to the way that the visual and vestibular systems integrate with one another. Pilots however, consistently show a preference for motion systems in flight simulation, which drives the need for finding a more economical instrument for providing motion cues. More research needs to be conducted on dynamic motion seats in order to determine whether or not they are the solution to this need. 


\section{References}

ACME Worldwide Enterprises Inc. (2014). VMax True Q Dynamic Motion Seats [Online Image]. Retrieved June 1, 2014 from http://www.acmeworldwide.com/dynamic_motion_seat_VMax.htm.

Adams, J. (1961). Some considerations in the design and use of dynamic flight simulators. Selected Papers on Human Factors in the Design and Use of Control Systems (Ed. H. W. Sinaiko), pp. 88-114. Dover Publications, Inc., N.Y.

Ashkenas, I. L. (1985). Collected flight and simulation comparisons and considerations. Paper presented at the Flight Mechanics Panel Symposium on Flight Simulation, Cambridge, United Kingdom.

Andersen, G. J. \& Braunstein, M. L. (1985). Induced self-motion in central vision. Journal of Experimental Psychology: Human Perception and Performance, $11,122-132$.

Angelaki, D., Gu, Y., \& DeAngelis, G. (2009). Multisensory integration: Psychophysics, neurophysiology, and computation. Current Opinion in Neurobiology, 19, 452458.

Bartel, C. \& Foster, D. (2004). Life cycle motion base cost comparison: Electric vs. hydraulic. SimTecT 2004: Simulation - Better Than Reality? Simulation Industry Association of Australia.

Beauchamp, M., Argall, B., Bodurka, J., Duyn, J., \& Martin, A. (2004). Unraveling multisensory integration: patchy organization within human STS multisensory cortex. Nature Neuroscience, 7, 1190-1192. 
Benevento, L.A., Fallon, J., Davis, B.J. \& Rezak, M. (1977). Auditory-visual interaction in single cells in the cortex of the superior temporal sulcus and the orbital frontal cortex of the macaque monkey. Journal of Experimental Neurology, 57, 849-872.

Berthoz, A., Pavard, B., \& Young, L. R. (1975). Perception of linear horizontal selfmotion induced by peripheral vision (linearvection) basic characteristics and visual-vestibular interactions. Experimental Brain Research, 23, 471-489.

Bird, D. (1963). Flight control studies in the small stick deflection area. Presented to AIAA Simulation for Aerospace Flight Conference, Aug. 26-28.

Blair, W., Kiemel, T., Jeka, J. J., \& Clark, J. E. (2012). Development of multisensory reweighting is impaired for quiet stance control in children with developmental coordination disorder (DCD). PLoS ONE, 7, 1-18.

Bottini, G., Sterzi, R., Paulesu, E., Vallar, G., Cappa, S. F., Erminio, F., et al. (1994). Identification of the central vestibular projections in man: A positron emission tomography activation study. Experimental Brain Research, 99, 164-169.

Brandt, T., Bartenstein, P., Janek, A., \& Dieterich, M. (1998). Reciprocal inhibitory visual-vestibular interaction. Visual motion stimulation deactivates the parietoinsular vestibular cortex. Brain, 121, 1749-1758.

Brandt, T., Dichgans, J., \& Koenig, E. (1973). Differential effects of the central versus peripheral vision on egocentric and exocentric motion perception. Experimental Brain Research, 16, 476-491.

Brandt, T. H., Dieterich, M., \& Danek, A. (1994). Vestibular cortex lesions affect the perception of verticality. Annals of Neurology, 35, 403-412. 
Brickman, B. J., Hettinger, L. J., \& Haas, M. W. (2000). Multisensory Interface Design for Complex Task Domains: Replacing Information Overload With Meaning in Tactical Crew Stations. The International Journal of Aviation Psychology, 10, 273-290.

Burki-Cohen, J., Sparko, A. L., \& Go, T. H. (2007). Training value of a fixed-base flight simulator with a dynamic seat. AIAA Modeling and Simulation Technologies Conference, 20-23 August 2007, AIAA-2007-6564.

Burki-Cohen, J., Sparko, A. L., Jo, Y. J., \& Go, T. H. (2009). Effects of visual, seat, and platform motion during flight simulator air transport pilot training and evaluation. Proceeding of the 15th International Symposium on Aviation Psychology, 27-30 April.

Bussolari, S. R., Young, L. R., \& Lee, A. T. (1988). The use of vestibular models for design and evaluation of flight simulator motion (AGARD CP-433). Neuilly Sur Seine, France: NATO Advisory Group for Aerospace Research and Development.

Calvert, G.A. (2001). Crossmodal processing in the human brain: insights from functional neuroimaging studies. Cerebral Cortex 11, 1110-1123.

Camis, M., \& Creed, R.S. (1930). The physiology of the vestibular apparatus. American Journal of the Medical Sciences, 180, 849.

Caro, P. W., Jolley, O.B., Isley, R. N., \& Wright, R. H. (1972). Determiningtraining device requirements in fixed-wing aviator training (Technical Report HumRRO- 72-11). Alexandria, VA: Human Resources Research Organization. 
Cian, C., Barraud, P.A., Paillard, A. C., Hidot, S., Denise, P., \& Ventre-Dominey, J. (2014). Otolith signals contribute to inter-individual differences in the perception of gravity-centered space. Experimental Brain Research, 232, 1037-1045.

Cohen, B., Maruta, J., \& Raphan, T. (2001). Orientation of the eyes to gravitoinertial acceleration. Annals of the New York Academy of Sciences, 942, 241-258.

Colby, C. L. \& Duhamel, J. R. (1991). Heterogeneity of extrastriate visual areas and multiple parietal areas in the macaque monkey. Neuropsychologia, 29, 517-537.

Cooper, G. (1963). The use of piloted flight simulators in take-off and landing research. North Atlantic Treaty Organization, Advisory group for Aeronautical Research and Development, Report 430.

Dichgans, J., Brandt, T. (1978). Visual-vestibular interaction: Effects on self-motion perception and postural control. Handbook of sensory physiology (Eds. R. Held, H.W. Leibowitz, H.L. Teuber), pp. 756-804. Springer, Berlin.

Driver, J., \& Noesselt, T. (2008). Multisensory interplay reveals crossmodal influences on "sensory-specific" brain regions, neural responses, and judgments. Neuron, 57, $11-23$.

Ebenholtz, S. M., Shebilske, W. (1975). The doll reflex: Ocular counter-rolling with head- body tilt in the median plane. Vision Research, 15, 713-17.

Ehrsson, H. H., Spence, C., \& Passingham, R. E. (2004). That's my hand! Activity in premotor cortex reflects feeling of ownership of a limb. Science, 305, 875-877.

Eriksson, L. (2009). Toward a visual flow integrated display format to combat pilot spatial disorientation. The International Journal Of Aviation Psychology, 20, 124. 
Farmer, E., van Rooij, J., Riemersma, J., Joma, P., \& Morall, J. (1999). Handbook of Simulator Based Training. Aldershot, Hampshire, UK: Ashgate.

Fetsch, C. R., DeAngelis, G. C., \& Angelaki, D. E. (2013). Bridging the gap between theories of sensory cue integration and the physiology of multisensory neurons. Nature reviews: Neuroscience, 14, 429-42.

Frens, M. A., van Opstal, A. J. \& van der Willigen, R. F. (1995). Spatial and temporal factors determine audio-visual interactions in human saccadic eye movements. Perception and Psychophysiology, 57, 802-816.

Gentile, G., Petkova, V., \& Ehrsson, H. (2011). Integration of visual and tactile signals from the hand in the human brain: An fmri study. Journal of Neurophysiology, 105, 910-922.

Goebel, R., \& van Atteveldt, N. (2009). Multisensory functional magnetic resonance imaging: a future perspective. Experimental Brain Research, 198, 153-164.

Graziano, M. S. (1999). Where is my arm? The relative role of vision and proprioception in the neuronal representation of limb position. Proceedings of the National Academy of Sciences of the United States of America, 96, 10418-10421.

Graziano, M. S., \& Gross, C, G. (1993). A bimodal map of space: somatosensory receptive fields in the macaque putamen with corresponding visual receptive fields. Experimental Brain Research, 97, 96-109.

Grieg, I. (1996). Evaluation of a multi-axis dynamic cueing seat for use in helicopter training devices. Control \& Simulation Department, Defence Research Agency, Bedford, UK Heintzman. 
Hall, J. R. (1989). The need for platform motion in modern piloted flight training simulators (Technical Memorandum FM 35). Bedford, UK: Royal Aerospace Establishment, Minister of Defence.

Harrar, V., \& Harris, L. R. (2007) Multimodal ternus: Visual, tactile, and visuo-tactile grouping in apparent motion. Perception, 36, 1455-1464.

Haward, D. (1910) The Sanders Teacher. Flight, 2, 1006-1007.

Hikosaka, K., Iwai, E., Saito, H. \& Tanaka, K. J. (1988). Polyresponse properties of neurons in the anterior bank of the caudal superior temporal sulcus of the Macaque monkey. Journal of Neurophysiology, 60, 1615-1637.

Hughes, H. C., Reuter-Lorenz, P. A., Nozawa, G. \& Fendrich R. (1994). Visual-auditory interactions in sensory-motor processing: Saccades versus manual responses. Journal of Experimental Psychology: Human Perception and Performance, 20, 131-153.

Holmes, N. P., Spence, C. (2005). Multisensory integration : Space, time and superadditivity. The superior colliculus generates and controls eye and head. Current Biology, 15, 762-764.

Hyvärinen, J. (1981). Regional distributions of functions in parietal association area 7 of the monkey. Brain Research, 206, 287-303.

Jarmasz, J., \& Hollands J. G. (2009). Confidence intervals in repeated-measures designs: The number of observations principle. Canadian Journal of Experimental Psychology, 63, 124-138.

Johansson, G. (1977). Studies on visual-perception of locomotion. Perception, 6, 365376. 
Kappé, B., Van Erp, J., \& Korteling, J. E. (1999). Effects of headslaved and peripheral displays on lane-keeping performance and spatial orientation. Human Factors, 41, 453-466.

Khan, S., \& Chang, R. (2013). Anatomy of the vestibular system: A review. Neurorehabilitation, 32, 437-443.

King, A., \& Calvert, G. (2001). Multisensory integration: Perceptual grouping by eye and ear. Current Biology, 11, 322-325.

Klyde, D. H., Lampton, A. K., Schulze, P. C., Alvarez, D., Johnson, R., \& Rowe, L. (2013). The real-flight approach to assess flight simulator force cueing fidelity. Paper presented at AIAA Atmospheric Flight Mechanics (AFM) Conference, Boston, MA.

Lackner, J., \& DiZio, P. (2005). Vestibular, proprioceptive, and haptic contributions to spatial orientation. Annual Review of Psychology, 56, 115-147.

Lam, T. M., Mulder, M., van Passen, M. M. (2007). Haptic interface for UAV collision avoidance. The International Journal of Aviation Psychology, 17, 167-195.

Lee, A. T., \& Bussolari, S. R. (1989). Flight simulator platform motion and air transport pilot training. Aviation, Space, and Environmental Medicine, 60, 136-140.

Lee, B., \& Myung, R. (2013). Attitude indicator design and reference frame effects on unusual attitude recoveries. The International Journal of Aviation Psychology, 23, 63-90.

Lintern, G., Thomley-Yates, K., Nelson, B., \& Roscoe, S. (1987). Content, variety, and augmentation of simulated visual scenes for teaching air-to-ground attack. Human Factors, 29, 45-59. 
Maran, N., \& Glavin, R. (2003). Low-to high-fidelity simulation - A continuum of medical education? Medical Education, 37, 22-28.

Mardirossian, V., Karmali, F., Merfeld, D. (2014). Thresholds for human perception of roll tilt motion: Patterns of variability based on visual, vestibular, and mixed cues. Otology and Neurotology.

Martin, E. L., \& Waag, W. L. (1978a). Contributions of platform motion to simulator training effectiveness: Study I-Basic contact. Brooks Air Force Base, TX: Air Force Human Resources Laboratory.

Martin, E. L., \& Waag, W. L. (1978b). Contributions of platform motion to simulator training effectiveness: Study 2-Aerobatics. Brooks Air Force Base, TX: Air Force Human Resources Laboratory.

Matthews, P. B. C. (1972). The mammalian muscle receptors and their central actions. London, England: Edward Arnold.

McCauley, M. E. (2006). Do helicopter training simulators need motion bases? United States Army Research Institute for the Behavioral and Social Sciences, Technical Report 1176, Arlington, VA.

McGraw-Hill Science \& Technology Encyclopedia. (2005). Vestibular system [Online image]. Retrieved May 13, 2014 from http://www.answers.com/topic/vestibularsystem-1.

Miller, E. F., Graybiel, A. (1962). Counterrolling of the human eyes produced by head tilt with respect to gravity. Acta Oto-laryngologica, 54, 479-501. 
Miller, R. (1953) Psychological considerations in the design of training equipment. Report no. WADC-TR-54-563, AD 71202. Wright Patterson Air Force Base, OH; Wright Air Development Center.

Moore, S. T., Hirasaki, E., Raphan, T., \& Cohen, B. (2001). The human vestibulo-ocular reflex during linear locomotion. Annals of the New York Academy of Sciences, 942, 139-147.

Morgan, M., DeAngelis, G., \& Angelaki, D. (2008). Multisensory integration in macaque visual cortex depends on cue reliability. Neuron, 59, 662-673.

Nataupsky, M., Waag, W. L., Weyer, D. C., McFadden, R. W., \&McDowell, E. (1979). Platform motion contributions to simulator training effectiveness: Study 3. Interaction of motion with fields of view (AFHRL-TR-79-25). Brooks Air Force Base, TX: Air Force Human Research Laboratory.

Nicholson, C., Townsend, B., Staples, A., Gamble, M., \& Herdman, C. M. (2013). Effects of motion cueing on an attitude recovery task. Paper presented at Proceedings of the $16^{\text {th }}$ International Symposium on Aviation Psychology, Dayton, $\mathrm{OH}$.

Nozawa, G., Reuter-Lorenz, P. A. \& Hughes, H. C. (1994). Parallel and serial processes in the human oculomotor system: bimodal integration and express saccades. Biological Cybernetics, 72, 19-34.

Oxford Dictionary of Sports Science and Medicine. (2007). Muscle spindle [Online image]. Retrieved June 1, 2014 from http://www.answers.com/topic/muscle-spindle 
Page, R. (2000). The SimTecT 2000 Organising and Technical Committee. Brief history of flight simulation, (Sydney, Austr alia).

Parrish, R. V., Houck, J. A., \& Martin, D. J. (1977). Empirical comparison of a fixedbase and a moving base simulation of a helicopter engaged in visually conducted slalom runs (NASA technical Note D-8424). Hampton, VA: NASA Langley Research Center.

Pasma, D. L., Grant, S. C., Gamble, M., Kruk, R. V., \& Herdman, C. M. (2011, September). Utility of Motion and Motion-Cueing to Support Simulated In-Flight Rotary-Wing Emergency Training. Paper presented at Proceedings of the Human Factors and Ergonomics Society Annual Meeting, Las Vegas, NV.

Proske, U., \& Gandevia, S. (2009). The kinaesthetic senses. The Journal of Physiology, $587,4139-4146$.

Proske, U., \& Gandevia, S. (2012). The proprioceptive senses: their roles in signaling body shape, body position and movement, and muscle force. Physiological Reviews, 92, 1651-1697.

Purves D, Augustine GJ, Fitzpatrick D, et al., editors. Neuroscience. 2nd edition. Sunderland (MA): Sinauer Associates; 2001. The Otolith Organs: The Utricle and Sacculus. Available from: http://www.ncbi.nlm.nih.gov/books/NBK10792/

Rabbitt, R. D., Damiano, E., R., \& Grant, J. W. (2004) Biomechanics of the Semicircular Canals and Otolith Organs. Springer Handbook of Auditory Research, 19, 153201.

Regan, D. (1994). Spatial orientation in aviation: Visual contributions. Journal of Vestibular Research: Equilibrium \& Orientation, 5, 455-471. 
Reid, L. D., \& Nahon, M. A. (1988). Response of airline pilots to variations inflight simulator motion algorithms. Journal of Aircraft, 25, 639-646.

Richard, G., \& Parrish, R. (1984). Pilot differences and motion cuing effects on simulated helicopter roll. Human Factors, 26, 249-256.

Richer, F., Martinez, M., Cohen, H., \& Saint-Hilaire, J. M. (1991). Visual motion perception from stimulation of the human medial parieto-occipital cortex. Experimental Brain Research, 87, 649-652.

Riecke, B. E. (2010). Compelling self-motion through virtual environments without actual self-motion - Using self-motion illusions ('vection') to improve VR user experience. In J. Kim (Ed.), Virtual Reality. Shanghai, China: InTech.

Robinson, A., Mania, K., \& Perey, P. Flight simulation: Research challenges and user assessments of fidelity. London: CAE UK and University of Essex.

Rosen, K. (2008). The history of medical simulation. Journal of Critical Care, 23, 157166.

Sadowski, W., \& Stanney, K. (2002). Measuring and managing presence in virtual environments. In K. Stanney (Ed.), Handbook of virtual environments: Design, implementation, and applications. Lawrence Erlbaum Associates.

Salas, E., Cannon-Bowers, J. (2001). The science of training: A decade of progress. Annual Review of Psychology, 52, 471-499.

Schöne, H., \& Udo De Haes, H. (1968). Perception of gravity-vertical as a function of head and trunk position. Zeitschrift Für Vergleichende Physiologie, 60, 440-444. Sears, A., Jacko, J. (2009). Human-Computer interaction: Design issues, solutions, and applications. London, England: CRC Press. 
Seemungal, B. M., Guzman-Lopez, J., Arshad, Q., Schultz, S. R., Walsh, V., \& Yousif, N. (2013). Vestibular activation \& differentially modulates human early visual cortex and V5/MT excitability and response entropy. Cerebral Cortex, 23, 12-19.

Smedal, H., Rogers, T., Duane, T., Holden, G., \& Smith, J. (1963). The physiological limitations of performance during acceleration. Aerospace Medicine, 34, 48-55.

Stein, B. E., Huneycutt, W. S., \& Meredith, M. A. (1988). Neurons and behaviour: The same rules of multisensory integration apply. Brain Research, 448, 355-358.

Stein, B. E. \& Meredith, M. A. (1993). The Merging of the Senses (ed. Gazzaniga, M. S.) The MIT Press, Cambridge, Massachusetts.

Stein, B. E., \& Stanford, T. R. (2008). Multisensory integration: current issues from the perspective of the single neuron. Nature reviews: Neuroscience, 9, 255-66.

Sutton, D., Skelton, M., \& Holt, L. S. (2010). Application and implementation of dynamic motion seats. Interservice/Industry Training, Simulation, and Education Conference (I/ITSEC).

Tal, N., Amedi, A. (2009). Multisensory visual-tactile object related network in humans: Insights gained using a novel crossmodal adaptation approach. Experimental Brain Research, 198, 165-182.

Tannen, R. S., Nelson, W. T., Bolia, R. S., Base, W. A. F., Warm, J. S., \& Dember, W. N. (2004). Evaluating Adaptive Multisensory Displays for Target Localization in a Flight Task, The International Journal of Aviation Psychology, 14, 297-312.

Taylor, H., Lintern, G., Koonce, J., Kaiser, R., \& Morrison, G. (1991). Simulator scene detail and visual augmentation guidance in landing training for beginning 
pilots. Paper presented at the Aerospace Technology Conference and Exposition, Long Beach CA.

Taylor, H., Lintern, G., Koonce, J.,Kundle, D., Tschopp, J., \& Talleur, D. (1993). Scene content, field of view and amount of training in first officer training. In R.S. Jensen (Ed.), Proceedings of the $7^{\text {th }}$ International Symposium on Aviation Psychology (pp. 753-757). Columbus: Ohio State University.

Telford, L., Seidman, S.H, \& Paige, G.D. (1997). Dynamics of squirrel monkey linear vestibuloocular reflex and interactions with fixation distance. Journal of Neurophysiology, 78, 1775-1790.

United States Government. (2004). Vestibular system’s semicircular canal - a crosssection [Online image]. Retrieved May 13, 2014 from http://www.cami.jccbi.gov/aam-400/phys intro.htm.

Van Beuzekom, A. D., \& Van Gisbergen, J. A. M. (2000). Properties of the internal representation of gravity inferred from spatial-direction and body-tilt estimates. Journal of Neurophysiology, 84, 11-27.

Wallace, M. T., Meredith, M. A, \& Stein, B. E. (1998). Multisensory integration in the superior colliculus of the alert cat. Journal of neurophysiology, 80, 1006-1010.

Warren, W., \& Kurtz, K. (1992). The role of central and peripheral vision in perceiving the direction of self-motion. Perception \& Psychophysics, 51, 443-454.

Wickens, C. D., Small, R. L., Andre, T., Bagnall, T., \& Brenaman, C. (2008). Multisensory Enhancement of Command Displays for Unusual Attitude Recovery. The International Journal of Aviation Psychology, 18, 255-267. 
Wilson, V., \& Melvill Jones, G. (1979). Mammalian Vestibular Physiology. New York, USA: Premium Press.

Wong, S. C. P., \& Frost, B. J. (1978). Subjective motion and acceleration induced by the movement of the observer's entire visual field. Perception \& Psychophysics, 24, 115-120.

Woodruff, R. R., Smith, J. F., Fuller, J. R., \& Weyer, D. C. (1976). Full mission simulation in undergraduate pilot training: An exploratory study (AFHRL-TR76-84). Brooks Air Force Base, TX: Air Force Human Research Laboratory. 


\section{Appendix A - SONA Posting}

Study Name: The Role of Vision in the Perception of Vestibular Motion

Experimenter's Name: Ben Townsend, Masters Student, Department of Psychology

Experimenter's Phone: 613-520-2600 ext. 2487

Experiment Location: VSIM Building, room 2210

Description: This experiment will assess how visual information affects your ability to detect the magnitude of motion cues. Specifically, you will be secured in a motion-cueing seat that simulates an aircraft banking at various angles. At the same time, a horizontal line displayed on a large-screen monitor will tilt left or right. Your task will be to judge the magnitude of the simulated bank angle and to determine whether or not the horizontal line tilt was consistent with the bank angle. In rare occasions, participants may experience nausea that is typically mild (no vomiting) and lasts for less than 5 minutes once the simulation ends. This study has received clearance by the Carleton University Psychology Research Ethics Board (Ethics Approval: 14-048).

Eligibility Requirements: Normal or corrected-to-normal visual acuity

Duration: 1.0 hour

Percentage: 1.0 Percentage

Preparation: None 


\title{
Informed Consent Form
}

\section{Appendix B - Informed Consent Form}

\begin{abstract}
Study: The Role of Vision in the Perception of Vestibular Motion
Faculty Sponsor: Dr. Chris Herdman, Department of Psychology, Carleton University, tel. 520$2600 \times 8122$

The purpose of this informed consent form is to ensure that you understand both the purpose of the study and the nature of your participation. The informed consent must provide you with enough information so that you have the opportunity to determine whether you wish to participate in the study. This study has received clearance by the Carleton University Psychology Research Ethics Board (Ethics Approval: 14-048). Please ask the researcher to clarify any concerns that you may have after reading this form.
\end{abstract}

Research Personnel: The following people are involved in this research and may be contacted at any time should you require further information about this study:

\begin{tabular}{|c|c|c|c|c|}
\hline Name & Title & Department & Email & Phone \\
\hline Chris Herdman & Professor & Psychology & chris_herdman@carleton.ca & $520-2600 \times .8122$ \\
\hline Ben Townsend & MA Student & Psychology & ben_townsend@carleton.ca & $520-2600 \times .2487$ \\
\hline
\end{tabular}

Other Contacts: Should you have any ethical concerns regarding this study, please contact Dr. Shelley Brown. For any other concerns about this study, please contact Dr. Anne Bowker.

\begin{tabular}{|c|c|c|}
\hline Name & Position & Phone \\
\hline Dr. Shelley Brown & Chair, Psychology Research Ethics Board & $520-2600 \times .1505$ \\
\hline Dr. Anne Bowker & Chair, Department of Psychology & $520-2600 \times .8218$ \\
\hline
\end{tabular}

Purpose: Simulators are more commonly being used to train vehicle operators (pilots, drivers) because they offer a cost-effective alternative to real-world training. Many simulation environments now use motion and/or motion cueing to enhance the realism of the simulation. However, given that our visual system is dominant (i.e., if our eyes tell us that we are moving, then we believe that we are moving), it is not clear that motion is critical for improving simulation-based operator training. The purpose of this study is therefore to assess the extent to which the visual system plays a role in perceiving simulation-based motion.

Task: In this study, you will be seated and secured in a motion seat positioned in front of a largescreen monitor. A horizon line will appear on the monitor and will tilt either to the left or the right. The line tilt will be accompanied by a motion cue from the seat that will make you feel like you are tilting either left or right by varying degrees. Your task is to judge the magnitude of the motion cue and to determine whether the tilted horizon line was consistent or inconsistent with the motion cue. In some cases, the horizontal line will not be visible, in which case you will be asked to only judge the magnitude of the motion cue. All responses will be made using a button 
box. You will have unlimited time to respond, at which point the next trial will begin. The experiment will begin with some practice trials to help you become familiar with the motion seat and the various magnitudes of simulated tilt. The experiment consists of 3 blocks of 48 trials each, for a total of 144 experimental trials. Breaks will be provided between each block of trials.

Duration, Locale \& Compensation: Testing will take place in VSIM 2210 and will take approximately one hour. You will receive $1.0 \%$ credits for your participation.

Potential Risks/Discomfort: It is possible that you may experience mild nausea during this experiment. In the unlikely event that this occurs, please inform the experimenter immediately. The experiment will be terminated and you will be asked to close your eyes to alleviate the nausea. You will be given full credit even if you cannot continue the experiment. Please note that your performance on the task in this experiment does not provide an indication of your suitability for university studies. However, if you feel anxious and/or uncomfortable about your performance, then please bring your concerns to the researcher's attention immediately.

Anonymity/Confidentiality: All data collected in this experiment will be kept strictly confidential through the assignment of a coded number and securely stored on a local computer for a maximum of ten years. Similarly, this Informed Consent form will be kept for a maximum of ten years before being destroyed. The information provided will be used for research purposes only. You will not be identified by name in any reports produced from this study. Further, the information is made available only to the researchers associated with this experiment.

Right to Withdraw/Omit: You have the right to withdraw from this experiment at any time without academic penalty. If you wish to do so, you will still receive full credit. Your participation in this experiment is completely voluntary.

I have read the above description of the study assessing the role of vision in the perception of vestibular motion. By signing below, this indicates that I agree to participate in the study, and this in no way constitutes a waiver of my rights.

Name:

Date:

Signature:

Witness: 


\section{Appendix C - Debriefing Form}

\section{Debriefing}

\section{The Role of Vision in the Perception of Vestibular Motion}

Thank you for your participation. The study you have just participated in is designed to investigate the extent to which your visual system plays a role in perceiving vestibular motion. Previous research shows that motion cues tend to enhance performance in simulation-based training. However, the association between motion fidelity (how realistic the cue is) and performance has not been strongly established. One reason why motion may not be that important in simulation-based training is because our visual system tends to dominate over other sensory systems. In other words, if our eyes "tell" us that we are moving, then we believe we are moving - even if our vestibular system (responsible for detection motion) tells us that we are completely stationary. If the visual system plays a dominant role in perceiving motion, then it is possible that motion cueing is only providing redundant information and is therefore not essential for simulationbased training. It is hypothesized that inconsistent visual stimuli will decrease one's ability to accurately perceive self-motion. If you are interested in learning more about how vestibular motion cues affect performance in simulated environments, then please see the following:

Grant, P. R., Yam, B., Hosman, R., \& Schroeder, J. A. (2006). Effects of simulator motion on pilot behavior and perception. Journal of Aircraft, 43, 1914 - 1924.

Harris, L., Jenkin, M., \& Zikovitz, D. C. (1999). Vestibular cues and virtual environments: Choosing the magnitude of the vestibular cue. Virtual Reality, 229 $-236$.

This study has received clearance by the Carleton University Psychology Research Ethics Board (Ethics Approval: 14-048). Should you have any ethical concerns regarding this study then please contact Dr. Shelley Brown (Chair, Psychology Research Ethics Board, 613-520-2600, ext. 1505). Should you have any other concerns about this study then please contact Dr. Anne Bowker, (Chair, Department of Psychology, 613-520-2600 ext. 8218 ) or any of the following individuals: 


\begin{tabular}{|c|c|c|c|c|}
\hline$\underline{\text { Name }}$ & Title & Department & $\underline{\text { Study Role }}$ & Contact Info. \\
\hline Ben Townsend & MA Student & Psychology & Principal & R20-2600 x. \\
& & & Researcher & 2487 \\
\hline $\begin{array}{c}\text { Dr. Chris } \\
\text { Herdman }\end{array}$ & Professor & Psychology & Faculty Advisor & $520-2600 \mathrm{x}$. \\
& & & 8122 \\
\hline
\end{tabular}

